\title{
CARACTERIZACIÓN MORFOLÓGICA Y MOLECULAR DE GENOTIPOS MEJORADOS DE CAMOTE (Ipomoea batatas L.) PARA ECOSISTEMAS ÁRIDO-SALINO-BÓRICOS
}

\author{
René Chávez ${ }^{1}$, Pedro Gallo ${ }^{2}$, Genoveva Rossel ${ }^{3}$, Daniel Reynoso ${ }^{3}$, Humberto Leva 4, Nadia Vera ${ }^{5}$
}

\begin{abstract}
RESUMEN
El camote (Ipomoea batatas $\boldsymbol{L}$ ) es un cultivo de importancia agronómica y social por sus múltiples aplicaciones para la alimentación humana, agroindustria y como forraje para el ganado, por lo que resulta de gran interés generar nuevos genotipos superiores, que contribuyan a combatir el hambre o la desnutrición en las zonas de mayor necesidad del Tercer Mundo.

En el presente trabajo de investigación se han caracterizado a clones élites y variedades mejoradas, adaptadas a las condiciones árido-salino-bóricas de la costa del Pacífico Sur de Sudamérica.

El proceso se inició con una caracterización morfológica del follaje y de las raíces reservantes de los genotipos en estudio; luego, una evaluación de sus principales atributos agronómicos, su reacción frente al Virus del Moteado Plumoso del Camote (SPFMY) bajo condiciones de campo, así como su rendimiento, tolerancia al frio y resistencia a otras condiciones de estrés abiótico, cultivados en suelos árido-salino-bóricos. Finalmente, se realizó una caracterización molecular mediante la técnica del AFLP. Estos datos fueron analizados con el software NTSYS, obteniendo dendogramas en los que se demostró que todos los genotipos tienen un coeficiente de similaridad menor a 1 (uno), lo que significa que los genotipos en estudio son diferentes y distintivos, lográndose diferenciar claramente entre ellos, mostrando una gran diversidad genética interclonal. Asimismo, estos resultados brindan información que puede ser usada para los registros internacionales de patentes de clones y variedades mejoradas
\end{abstract}

Palabras claves: camote, Ipomoea batatas, caracterización morfológica y molecular, ecosistema árido - salino - bórico y adaptación.

\section{A BS TRACT}

The sweetpotato (Ipomoea batatas L.) is one of the most important crops in the world grown in the tropical and sustropical zones as a food crop, fodder and row material for agroindustry. This justifies the establishment of bredding programs to genere new superior genotypes with high yield and nutritional qualities.

This research involves the characterization of elite clones and improved sweetpotato varieties adapted to arid, saline and boric soil conditions of the pacific coastal desert for South America. The main morphological characterization has been carried out on foliage features, starchy roots, agronomic atributes, field reaction to the sweetpotato feathery mottle virus (SPFMV) aswed as yield, cold tolerance and betacarotene content. Molecular characterization has been focused on AFLP which data were analyzed using the software NTSYS to obtain dendrograms and interclonal similarities. Results from morphological and molecular characterization shows great interclonal genetic diversity of the sweetpotato germplasm evaluated aswed as a veery low degree of similarities under AFLP analysis. The whole characterization data could be used for fingerprinting and patentig these new superior sweetpotato genotypes.

KEY WORDS : sweetpotato, Ipomoea batatas, molecular and morphological characterization; arid, saline and boric agroecosystems, adaptation.

(1) Doctor en Genética de plantas. Universidad Nacional "Jorge Basadre Grohmann", Tacna - Perú

(2) Facultad de Agronomía, Universidad de Tarapacá Arica - Chile

(3) Unidad de Biotecnologia, Centro Internacional de la Papa, Lima-Peru

(4) Bach. en Ciencias Biológicas. Universidad Nacional "Jorge Basadre Grohmann", Tacna - Perú

(5) Bióloga - Microbióloga. Universidad Nacional "Jorge Basadre Grohmann", Tacna - Perú 


\section{INTRODUCCIÓN}

El camote, Ipomoea batatas $L$, es una de las especies de la familia Convolvulaceae, cultivada para la alimentación humana y animal en todos los países tropicales y subtropicales del mundo. Sus raíces reservantes presentan gran contenido de azúcares, caroteno y vitaminas, los cuales constituyen un alimento y una importante materia prima para la industria del almidón y del alcohol, así como un follaje muy apreciado.

El camote es cultivado en más de 100 paises y representa el tubérculo más importante en el Tercer Mundo después de la papa. A nivel mundial, Asia y Oceanía poseen el $80 \%$ del cultivo de camote, siendo la China el primer productor de camote con los más altos rendimientos por hectárea (Chávez, 1990). Los últimos datos en cuanto a esto último señalan una producción de $19827 \mathrm{Kg} . / \mathrm{Ha}$ (Chávez, 2002).

EI CIP y FAO, en una serie de textos señalan que el camote es una planta nústica tolerante a un amplio rango de condiciones edáficas y climáticas, lo que lo hace importante desde el punto de vista agrícola, industrial y alimenticio. Asimismo, señalan que la alta concentración de sales de los suelos árido-salinos del mundo son una limitante para la explotación masiva de plantas cultivadas, pero, sin duda, se puede trabajar con lavados de suelos y riego tecnificado permanente, labor que resulta inalcanzable por sus altos costos para los pequeños agricultores de los paises en desarrollo como el nuestro.

La manutención y ampliación de las áreas productivas de camote, Ipomoea batatas $L$., como también el aumento de los rendimientos fisicos son los principales objetivos de los programas de investigación y desarrollo de organismos internacionales y de los palses que poseen dicho cultivo. Por lo tanto, la individualización y selección de nuevas variedades con una más amplia adaptabilidad mayor diversidad, además excelentes características agronómicas y que respondan a los requerimientos de los agricultores y consumidores, contribuirán al cumplimiento de los objetivos trazados por cada institución.

Para el caso de zonas áridas, la incorporación y utilización de variedades resistentes a estreses abióticos se presentan como una buena alternativa para utilizar suelos árido-salinos en valles, pampas y áreas costeras del Pacífico Sur en Sudamérica. Distintas investigaciones realizadas en estos últimos diez años respecto a la resistencia del camote a varias problemáticas, donde encontramos la salinidad, presencia de boro, nemátodos y sequia, revelan que las plantas genéticamente mejoradas son más tolerantes a los suelos áridossalinos, lo cual permite potenciar muchas más grandes superficies del mundo, permitiendo con ello establecer cultivos e incorporar suelos que hoy se clasifican como áreas marginales a la producción, que no son utilizadas a la fecha (Chávez, 2002).

La gran importancia nutricional, industrial y agroecológica que ha adquirido el camote en la actualidad lleva a los fitomejoradores a buscar nuevas variedades que se adapten a condiciones adversas de cultivo como ser la aridez, salinidad y toxicidad por boro.

Los recursos genéticos deben ser evaluados para emplearlos eficientemente en la creación de material promisorio. Se necesita tener mayor información acerca de las características morfológicas de la planta, su asociación con el rendimiento y la influencia del ambiente. El rendimiento es afectado significativamente por las interacciones entre el genotipo y el ambiente. La selección inicial es un paso previo en el mejoramiento genético del camote, para la selección de clones con el objeto de incrementar el potencial y la estabilidad de rendimiento, así como incorporar atributos especificos de precocidad, calidad y resistencia a factores adversos bióticos (insectos, hongos, nemátodos) y abióticos (salinidad, sequia, boro) (Arévalo, 1995).

Se señala que luego de iniciado el convenio entre la Universidad Nacional de Tacna y el Centro Intemacional de la Papa (CIP) en nuestro pais, en el año 1985, se han dado pie a diversos trabajos de investigación entre los que destacan los de evaluaciones de respuestas fenotipicas de plantas tuberiferas a estreses abióticos más comunes de las zonas árido-salinas (Chávez, 1995).

El objetivo del presente trabajo es comparar los caracteres morfológicos y moleculares; y determinar las respuestas fenotipicas del germoplasma de camote mejorado en ecosistemas árido-salino-bóricos, estableciendo de esta manera la variabilidad genética entre los clones estudiados.

En este trabajo, más allá de determinar la variabilidad genética entre los clones, los resultados obtenidos servirán para seleccionar aquellos clones con betacaroteno en sus composiciones químicas, adaptados a las condiciones adversas propias de los suelos árido-salino-bóricos, con la finalidad de promover su cultivo masivo posteriormente.

\section{MATERIAL Y MÉTODOS}

\section{Ubicación del Campo Experimental:}

La multiplicación in vitro de los genotipos se realizó en las instalaciones del Laboratorio de Cultivos in Vitro de la Facultad de Ciencias Agricolas de la Universidad Nacional Jorge Basadre Grohmann de Tacna.

La multiplicación acelerada de los genotipos y la caracterización morfológica preliminar de los mismos se realizb en un invernadero construido en las afueras 
del referido Laboratorio.

La evaluación en campo de los genotipos en estudio se realizó en el Centro Experimental Agrícola La Yarada CEA - II, perteneciente a la Universidad Nacional Jorge Basadre Grohmann de Tacna.

\section{Material Experimental:}

En una primera etapa se evaluaron los siguientes genotipos:

\begin{tabular}{|l|l|}
\hline 1. Costanero & 14. TN 92.024 \\
\hline 2. Salyboro & 15. TN 95.549 \\
\hline 3. Comensal & 16. TN 89.021 \\
\hline 4. Tacna & 17. TN 95.633 \\
\hline 5. Nacional & 18. TN 95.195 \\
\hline 6. Caplina & 19. TN 95.119 \\
\hline 7. Atacama & 20. TN 95.517 \\
\hline 8. Yarada & 21. TN 95.031 \\
\hline 9. TN 95.075 & 22. TN 95.537 \\
\hline 10. TN 95.667 & 23. TN 94.060 \\
\hline 11. TN 91.012 & 24. TN 95.126 \\
\hline 12. TN 95.065 & 25. TN 95.032 \\
\hline 13. TN 95.596 & \\
\hline
\end{tabular}

En una segunda etapa se evaluaron los siguientes 17 genotipos chinos y 25 genotipos peruanos:

\section{GENOTIPOS DE CHINA POPULAR:}

\begin{tabular}{|c|c|c|c|}
\hline & \multicolumn{2}{|c|}{} & \\
\hline 440024 & Yaru Shu 1 & 440158 & Chao Shu 1 \\
\hline 440025 & Xushu 18 & 440238 & Fong Su N 1 \\
\hline 440027 & Ning Shu 1 & 440385 & Bei Jieng 553 \\
\hline 440030 & Nong Da Hong & 440386 & Ziding V100 \\
\hline 440151 & Laio Shu 44 & 440387 & Xindazi \\
\hline 440153 & Ji 76115 & 440388 & Ghishu 2 \\
\hline 440155 & Yu Bei Bai & 440389 & Xiangza 9 \\
\hline 440156 & Guang Shu 16 & 440426 & Fong Sow Bai \\
\hline 440157 & Ning Shu 2 & & \\
\hline
\end{tabular}

\section{GENOTIPOS DE PERÚ:}

\begin{tabular}{|l|l|}
\hline TN 89.021 & TN 95.195 \\
\hline TN 91.012 & TN 95.324 \\
\hline TN 92.024 & TN 95.507 \\
\hline TN 92.091 & TN 95.514 \\
\hline TN 94.060 & TN 95.517 \\
\hline TN 95.031 & TN 95.537 \\
\hline TN 95.032 & TN 95.549 \\
\hline TN 95.041 & TN 95.599 \\
\hline TN 95.065 & TN 95.611 \\
\hline TN 95.075 & TN 95.649 \\
\hline TN 95.119 & TN 95.660 \\
\hline TN 95.122 & Var. Local \\
\hline TN 95.126 & \\
\hline
\end{tabular}

Finalmente se hizo una caracterización molecular de 10 genotipos, incluyendo el clon élite ST 87.030 y la variedad local Yema de Huevo.

\begin{tabular}{|l|l|}
\hline 1. Atacama & 6. Salyboro \\
2. Caplina & 7. ST 87.030 \\
3. Comensal & 8. Tacna \\
4. Costanero & 9. Yarada \\
5. Nacional & 10. Yema de Huevo (Tambeño) \\
\hline
\end{tabular}

\section{Métodos:}

\section{Conservación y Multiplicación del Germoplasma:}

En la primera parte del trabajo se realizó una micropropagación masiva in vitro de los clones en estudio, con la finalidad de conservar el germoplasma y de obtener la mayor cantidad de individuos que puedan ser sometidos posteriormente a las evaluaciones finales.

En la segunda parte del trabajo se trasladaron los individuos obtenidos en el laboratorio al invernadero donde fueron sometidos a métodos de multiplicación rápida por esquejes, para luego pasar a la etapa de caracterización. Esta multiplicación por esquejes permite contar con material de una alta sanidad y asegurar que los materiales correspondan a la variedad en proceso de multiplicación (Hidalgo, 1997).

\section{Evaluación del Material Experimental:}

La caracterización morfológica correspondió a la segunda parte del proyecto luego de ser multiplicado cada uno de los genotipos.

La caracterización morfológica se realizó basándose en descriptores establecidos por el Centro Internacional de la Papa y que se presentan en las tablas $\mathrm{N}^{\circ} 01 \mathrm{y}$ 02. Consistio en una descripción de los siguientes aspectos: características de la Hoja Madura (color, forma, tipo y número de lóbulos). Asimismo, las características de la Raíz Reservante (color de la piel, color principal de la pulpa, color secundario de la pulpa y su distribución, y finalmente el contenido de betacaroteno).

Se hizo la tercera parte consistente en la Evaluación Experimental bajo condiciones de campo en suelos árido-salino-bóricos, para lo cual los clones fueron llevados al CEA II. Se evaluaron las siguientes RESPUESTAS FENOTÍPICAS DE LOS CLONES A ESTRESES ABIOTICOS:

1.Capacidad de prendimiento bajo condiciones de Invierno y Verano.

2.Vigor del follaje a los 120 dias. 


\section{Grado de tuberización (performancia agronómica).}

Para la Evaluación se utilizó la escala de grados 1-3-5-7-9, la cual es recomendada por el Centro Internacional de la Papa (CIP).

Para la caracterización molecular de los ciones se siguieron los pasos que a continuación se detallan:

\section{Extracción de ADN}

2. Caracterización molecular con el Marcador AFLP (Método Blecker et al. 1997), lo cual consistió en 04 procesos: a) Digestión del ADN; b) Ligación de los adaptadores; c) Preamplificación de los fragmentos digeridos; d)Amplificación selectiva de los fragmentos digeridos.

3. Tinción Silver Sequence de los geles (método propuesto por el Technical Manual Promega, 1999).

4. Lectura de los geles.

\section{Diseño Experimental:}

El diseño elaborado para este trabajo consistió en bloques completamente aleatorios con tres repeticiones y 42 tratamientos.

Para el análisis estadístico se usó la técnica del análisis de Varianza. Se empleó la Prueba de $F$ de 0,05 y 0,01 de probabilidad. Para las comparaciones múitiples medias entre los tratamientos en estudio se utilizó la Prueba de significación de Duncan.

Para la tabulación de datos expresados en porcentajes se hizo necesario transformar datos utilizando la siguiente fórmula: Arcseno $\sqrt{x}$; donde $\mathrm{X}$ es el dato a transformar (Steel y Torrie).

Para el análisis de datos no paramétricos se utilizó la Prueba de Friedmann, según la fórmula:

$$
\begin{array}{ll}
\mathrm{Xr}^{2}=\frac{12}{\mathrm{bt}(\mathrm{t}+1)} \Sigma \mathrm{r}^{2}-3 b(\mathrm{t}+1) \\
\text { donde: } & \\
\mathrm{b} & : \text { Número de repeticiones } \\
\mathrm{t} & : \text { Número de tratamientos } \\
\mathrm{ri}^{2} & : \text { Suma de rangos } \\
12 \text { y } 3 & : \text { constantes }
\end{array}
$$

\section{RESULTADOS}

En las páginas siguientes, se detallan sendos cuadros en los que se describen los caracteres morfológicos y moleculares de los genotipos en estudio. 
Cuadro $N^{\circ} 01$ : Caracterización mortologica de genotipos mejorados de camote de origen peruano y chino cultivedos en suelos árido-salino-boriccos La Yarada, $2001-2003$

\begin{tabular}{|c|c|c|c|c|c|c|c|c|c|}
\hline \multirow[b]{2}{*}{$N^{r}$} & \multirow[b]{2}{*}{ Clon } & \multicolumn{7}{|c|}{ Caracterización morfológica } & \multirow[b]{2}{*}{$\begin{array}{c}\text { Regltencla } \\
\text { a VIrus } \\
\text { SPFMV }\end{array}$} \\
\hline & & Forma hoja & TIpo Lobulo & Número Lob. & Color de Piel & $\begin{array}{c}\text { Color Princ. } \\
\text { Pulpa }\end{array}$ & $\begin{array}{c}\text { Color Secund. } \\
\text { Puipa }\end{array}$ & $\begin{array}{l}\text { Distribuc.color } \\
\text { se cund. Puipa }\end{array}$ & \\
\hline 1 & Costanero & 3 & 1 & 1 & 8 & 8 & 9 & 1 & + \\
\hline 2 & Salyboro & 6 & 7 & 3 & 4 & 8 & 0 & 0 & + \\
\hline 3 & Comensal & 6 & 7 & 5 & 5 & 6 & 0 & 0 & + \\
\hline 4 & Tacna & 6 & 7 & 5 & 2 & 3 & 9 & 3 & - \\
\hline 5 & Naclonal & 6 & 3 & 3 & 5 & 1 & 0 & 0 & - \\
\hline 6 & Capllna & 6 & 3 & 3 & 2 & 2 & 0 & 0 & - \\
\hline 7 & Atacama & 6 & 7 & 5 & 9 & 2 & $\theta$ & 1 & $\therefore$ \\
\hline 8 & Yarada & 6 & 7 & 5 & 9 & 2 & 9 & 1 & + \\
\hline 9 & TN 89.021 & 8 & 7 & 3 & 2 & 5 & 4 & 2 & + \\
\hline 10 & TN 91.012 & 3 & 1 & 1 & 4 & 8 & 0. & 0 & + \\
\hline 11 & TN 92.091 & 3 & 0 & 1 & 2 & 2 & 2 & 4 & - \\
\hline 12 & TN 92.024 & 3 & 1 & 5 & 4 & 6 & 0 & 0 & + \\
\hline 13 & TN 93.122 & 6 & 3 & 3 & 2 & 2 & 8 & 3 & - \\
\hline 14 & TN 94.060 & 6 & 7 & 5 & 8 & 1 & 0 & 0 & + \\
\hline 15 & TN 95.031 & 3 & 1 & 1 & 5 & 3 & 9 & 3 & + \\
\hline 16 & TN 95.032 & 3 & 1 & 1 & 1 & 1 & 0 & 0 & + \\
\hline 17 & TN 95.041 & 6 & 5 & 5 & 8 & 7 & 3 & $4-3$ & - \\
\hline 18 & TN 95.065 & 6 & 3 & 3 & 4 & 7 & 0 & 0 & - \\
\hline 19 & TN 95.075 & 6 & 7 & 5 & 9 & 8 & 9 & 1 & - \\
\hline 20 & TN 95.119 & 6 & 7 & 5 & 9 & 3 & 9 & 7 & - \\
\hline 21 & TN 95.126 & 5 & 7 & 5 & 7 & 2 & 0 & 0 & + \\
\hline 22 & TN 95.195 & 5 & 1 & 3 & 9 & 3 & 9 & 1 & \pm \\
\hline 23 & TN 95.517 & 6 & 7 & 5 & 1 & 3 & 9 & 9 & - \\
\hline 24 & TN 95.537 & 5 & 3 & 3 & 2 & 3 & 0 & 0 & + \\
\hline 25 & TN 95.549 & 3 & 1 & 3 & 9 & 7 & 9 & 1 & + \\
\hline 28 & TN 95.696 & 5 & 7 & 5 & 6 & 7 & 0 & 0 & - \\
\hline 27 & TN 95.633 & 3 & 3 & 1 & 2 & 5 & 0 & 0 & - \\
\hline 28 & TN 95.649 & 6 & 7 & 5 & 4 & 6 & 8 & 8 & - \\
\hline 29 & TN 95.660 & 3 & 0 & 1 & 8 & 6 & 4 & 2 & - \\
\hline 30 & TN 95.667 & 3 & 1 & 1 & 9 & 7 & 9 & 7 & - \\
\hline 31 & 440027 & 3 & 0 & 1 & 7 & 5 & 0 & 0 & - \\
\hline 32 & 440151 & 6 & 7 & 5 & 6 & 3 & 3 & 1 & - \\
\hline 33 & 440158 & 6 & 5 & 5 & 2 & 1 & 0 & 0 & - \\
\hline 34 & 440385 & 3 & 0 & 1 & 7 & 2 & 0 & 0 & - \\
\hline 36 & 440387 & 3 & 0 & 1 & 8 & 2 & 0 & 0 & - \\
\hline 36 & 440388 & 3 & 0 & 1 & 8 & 2 & 0 & 0 & - \\
\hline 37 & 440389 & 3 & 0 & 1 & 6 & 4 & 4 & 4 & - \\
\hline
\end{tabular}

La evaluación de resistencia a vins fue hecha en campo, y se muestran los resultados de tres afos de experiencla. El signo más (+) muestra la susceptibilidac

es decir, la presencia de sintomas; y el signo menos $(-)$ representa la resistencia o ausencia de sintomas.

Cua dro $\mathrm{N}^{\bullet} 02$ : Rendimiento bajo condiciones de verano e invierno en suelos érido-salino-bóricos de genotipos mejorados de camote de origen peruano y chino. La Yarade, 2001-2003

\begin{tabular}{|c|c|c|c|c|c|c|c|c|c|c|c|}
\hline \multicolumn{6}{|c|}{ CONDKIONES DE VERANO } & \multicolumn{6}{|c|}{ CONDICIONES DE INVIERNO } \\
\hline $\mathbf{N}^{*}$ & Clon & $\begin{array}{c}\text { Capac. } \\
\text { Prendimiento }\end{array}$ & $\begin{array}{l}\text { Vigor } \\
\text { Foliaje }\end{array}$ & $\begin{array}{l}\text { Grado } \\
\text { Tuberiz. }\end{array}$ & Betacaroteno & $N$ & Clon & $\begin{array}{c}\text { Capac. } \\
\text { Prendimlento }\end{array}$ & $\begin{array}{l}\text { Vigor } \\
\text { Follaje }\end{array}$ & $\begin{array}{c}\text { Grado } \\
\text { Tuberlx. }\end{array}$ & Betacaroteno \\
\hline 1 & Costanero & 9 & 9 & 7 & 9 & 1 & TN 89.021 & 8 & 7 & 1 & - \\
\hline 2 & Salyboro & 7 & 9 & 9 & 9 & 2 & $\mathrm{TN} 92.024$ & 7 & 5 & 3 & - \\
\hline 3 & Comensal & 9 & 7 & 9 & 7 & 3 & TN 92.091 & 7 & 5 & 3 & 1 \\
\hline 4 & Tacna & 7 & 9 & 7 & 3 & 4 & $\pi N 93.122$ & 7 & 7 & 3 & 1 \\
\hline 5 & Naclonal & 7 & 7 & 5 & 1 & 5 & TN 94.060 & 7 & 5 & 3 & - \\
\hline 6 & Capilina & 9 & 9 & 8 & 1 & 6 & TN 95.031 & 5 & 7 & 1 & - \\
\hline 7 & Atacama & 9 & 9 & 7 & 1 & 7 & TN 95.032 & 7 & 7 & 1 & - \\
\hline 8 & Yarada & 7 & 9 & 9 & 1 & 8 & TN 95.041 & 7 & 7 & 8 & 7 \\
\hline 8 & Yema de Huevo & 8 & 9 & 3 & 3 & 2 & TN 95.055 & 9 & 9 & 5 & - \\
\hline 10 & TN 89.021 & 7 & 7 & 7 & 5 & 10 & TN 95.076 & 7 & 7 & 7 & 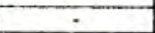 \\
\hline 11 & TN 91.012 & 8 & 7 & 9 & 9 & 11 & TN 95.119 & 9 & 9 & 3 & - \\
\hline 12 & TN 82.024 & 7 & 5 & 7 & 7 & 12 & TN 95.195 & 7 & 7 & 3 & - \\
\hline 13 & TN 94.060 & 7 & 9 & 9 & 1 & 13 & TN 96.549 & 7 & 5 & 5 & - \\
\hline 14 & TN 95.031 & 5 & 7 & 7 & 3 & 14 & TN 96.598 & 7 & 7 & 1 & - \\
\hline 15 & TN 95.032 & 5 & 7 & 9 & 1 & 15 & TN 95.649 & 9 & 7 & 7 & 9 \\
\hline 16 & TN 96.085 & 9 & 7 & 9 & 7 & 18 & TN 95.660 & 9 & 9 & 3 & 5 \\
\hline 17 & TN 95.075 & 9 & 9 & 9 & 9 & 17 & Var. Local & 5 & 7 & 3 & 3 \\
\hline 18 & TN 95.119 & 7 & 5 & 7 & 3 & 18 & TN 95.614 & 5 & 5 & 1 & - \\
\hline 19 & TN 95.126 & 7 & 7 & 9 & 1 & 10 & TN 95.126 & 1 & 1 & 1 & - \\
\hline 20 & TN 85.195 & 9 & 8 & 9 & 3 & 20 & TN 95.324 & 1 & 1 & 1 & $\therefore$ \\
\hline 21 & TN 95.517 & 9 & 5 & 9 & 3 & 21 & TN 95.507 & 1 & 1 & 1 & 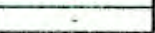 \\
\hline 22 & TN 95.637 & 8 & 7 & 7 & 3 & 22 & TN 91.012 & 1 & 1 & 1 & - \\
\hline 23 & TN 95.549 & 9 & 5 & 9 & 7 & 23 & TN 95.517 & 1 & 1 & 1 & . \\
\hline 24 & TN 95.596 & 9 & 7 & 9 & 7 & 24 & TN 86.537 & 1 & 1 & 1 & $\cdot$ \\
\hline 25 & TN 85.633 & 7 & 5 & 7 & 5 & 25 & TN 96.611 & 1 & 1 & 1 & - \\
\hline 26 & TN 95.687 & 9 & 9 & 5 & 7 & 28 & 440027 & 7 & 7 & 5 & . \\
\hline & & & & & & 27 & 440151 & 3 & 3 & 1 & 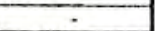 \\
\hline & & & & & & 28 & 440158 & 5 & 5 & 1 & $\cdot$ \\
\hline & & & & & & 29 & 440385 & 5 & 5 & 1 & - \\
\hline & & & & & & 30 & 440387 & 7 & 7 & 1 & - \\
\hline & & & & & & 31 & 440388 & 5 & 3 & 1 & $\therefore$ \\
\hline & & & & & & 32 & 440389 & 5 & 5 & 3 & $\therefore$ \\
\hline
\end{tabular}

Capacidad de Prendimiento, Vigor de Follaje, Grado de Tuberizacion y Contenido de Betacaroteno fueron svaluados según la escala del CIP: 1-3-5-7-9 Los que figuran con signo $(-)$ no fueron evaluado 
TABLA N 01: EXPLICACION DE LOS DESCRIPTORES PARA FOLLAJE

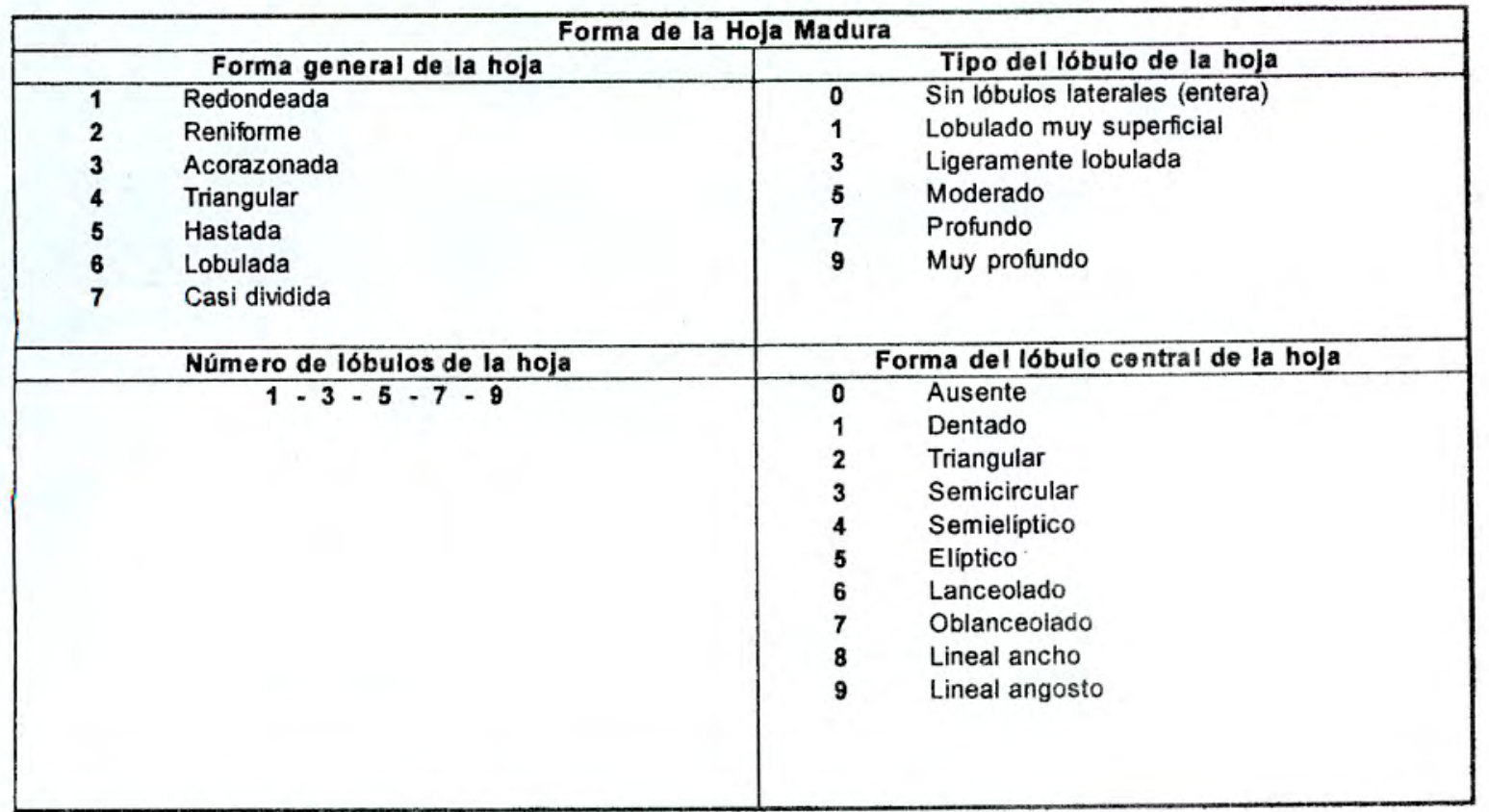

TABLA N02: EXPLICACIÓN DE LOS DESCRIPTORES PARA RAÍCES RESERVANTES

\begin{tabular}{|c|c|c|c|c|}
\hline & \multicolumn{2}{|c|}{$\begin{array}{l}\text { Color de la piel } \\
\text { Primer Número: Color principal de la piel A } \\
\text { Segundo Número: Color secundario de la piel B }\end{array}$} & & \\
\hline & \multicolumn{2}{|c|}{ A Color Principal } & \multicolumn{2}{|c|}{ B Color Secundario } \\
\hline & 1 & Blanco & 0 & Ausente \\
\hline & 2 & Crema & 1 & Blanco \\
\hline & 3 & Amarillo & 2 & Crema \\
\hline & 4 & Naranja & 3 & Amarillo \\
\hline & 5 & Naranja-marrón & 4 & Naranja \\
\hline & 6 & Rosado & 5 & Naranja- marrón \\
\hline & 7 & Rojo & 6 & Rosado \\
\hline & 8 & $\begin{array}{l}\text { Púrpura-rojo } \\
\text { Púrpura oscuro }\end{array}$ & 7 & Rojo \\
\hline \multicolumn{5}{|c|}{ Color de la pulpa } \\
\hline \multicolumn{3}{|c|}{ Color principal de la pulpa } & \multicolumn{2}{|c|}{ Distribución del color secundario } \\
\hline 1 & \multicolumn{2}{|l|}{ Blanco } & 0 & Ausente \\
\hline 2 & \multicolumn{2}{|l|}{ Crema } & 1 & Anillo delgado en la corteza \\
\hline 3 & \multicolumn{2}{|c|}{ Crema oscuro } & 2 & Anillo grueso en la corteza \\
\hline 4 & \multicolumn{2}{|c|}{ Amarillo pálido } & 3 & Manchas esparcidas en la pulpa \\
\hline 5 & \multicolumn{2}{|c|}{ Amarillo oscuro } & 4 & Mancha circular en el centro de la piel ( \\
\hline 6 & \multicolumn{2}{|c|}{ Naranja pálido } & 5 & Anillo delgado en la pulpa \\
\hline 7 & \multicolumn{2}{|c|}{ Naranja intermedio } & 6 & Anillo grueso en la pulpa \\
\hline 8 & \multirow{2}{*}{\multicolumn{2}{|c|}{$\begin{array}{l}\text { Naranja oscuro } \\
\text { Fuertemente pigmentado con antocianinas }\end{array}$}} & 7 & Anillo y otras áreas \\
\hline 9 & & & 8 & Color en secciones longitudinales \\
\hline \multicolumn{3}{|c|}{ Color secundario de la puipa } & $\begin{array}{c}9 \\
10\end{array}$ & $\begin{array}{l}\text { El color cubre la mayoría de la pulpa } \\
\text { El color cubre toda la pulpa }\end{array}$ \\
\hline 0 & \multicolumn{2}{|l|}{ Ausente } & \multicolumn{2}{|c|}{$\left({ }^{*}\right)$ Caracterís tica no reglstrada en el Manual deI CIP } \\
\hline 1 & \multicolumn{2}{|l|}{ Blanco } & & \\
\hline 2 & \multicolumn{2}{|l|}{ Crema } & & \\
\hline 3 & \multicolumn{2}{|l|}{ Amarillo } & & \\
\hline 4 & \multicolumn{2}{|l|}{ Naranja } & & \\
\hline 5 & \multicolumn{2}{|l|}{ Rosado } & & \\
\hline 6 & \multicolumn{2}{|c|}{ Rojo } & & \\
\hline 7 & \multicolumn{2}{|c|}{ Púrpura-rojo } & & \\
\hline 8 & \multicolumn{2}{|c|}{ Púrpura } & & \\
\hline 9 & \multicolumn{2}{|c|}{ Púrpura oscuro } & & \\
\hline
\end{tabular}


Cuadro $\mathrm{N}^{\circ} 03$ : Caracterización molecular. Resumen general de la caracterización de las bandas electroforéticas con AFI de los clones estudiados. Tacna, 2001

\begin{tabular}{|c|c|c|c|c|c|c|c|}
\hline No & 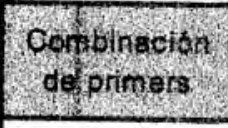 & $\begin{array}{l}\text { No Totals } \\
\text { band }\end{array}$ & $\begin{array}{l}\text { Nack } \\
\text { batc } \\
\text { Do }\end{array}$ & 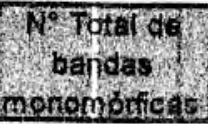 & $\begin{array}{l}N^{\circ} \text { total os } \\
\text { bagsas } 1 B=1\end{array}$ & $\begin{array}{l}\text { No total } 60 \\
\text { bandge 184s }\end{array}$ & 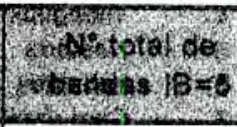 \\
\hline 1 & AGG/CAC & 26 & 20 & 6 & 11 & 8 & 7 \\
\hline 2 & ACC/CTG & 33 & 26 & 7 & 23 & 8 & 2 \\
\hline 3 & ACC/CAT & 43 & 34 & 9 & 17 & 19 & 7 \\
\hline 4 & ACG/CAC & 28 & 21 & 7 & 7 & 17 & 4 \\
\hline 5 & AAC/CTC & 29 & 28 & 1 & 3 & 20 & 6 \\
\hline 6 & $A G C / C A G$ & 23 & 15 & 8 & 14 & 5 & 4 \\
\hline 7 & AAC/CTG & 37 & 32 & 5 & 0 & 23 & 14 \\
\hline 8 & $\mathrm{ACC} / \mathrm{CTG}$ & 30 & 20 & 10 & 19 & 10 & 1 \\
\hline & TOTAL & 249 & 196 & 53 & 94 & 110 & 45 \\
\hline
\end{tabular}

En el cuadro anterior se encuentra el resumen de la caracterización de bandas electroforéticas con 8 combinaciones de primers usados, obteniendo un total de 249 bandas. 196 polimórficas y 53 monomórficas; esto quiere decir que utilizando las 8 combinaciones de primers existe un alto número de bandas polimórficas que ayudaron a diferenciar los genotipos de camote

CUADRO N 04 : Caracterización molecular. Porcentaje de bandas polimórficas y monomorficas, y grado de intensidad en el genomio de camote usando ocho combinaciones de primers. Tacna, 2001

\begin{tabular}{|c|c|c|c|c|c|c|c|}
\hline No: & $\begin{array}{l}\text { Gombineclon } \\
\text { deprimis }\end{array}$ & 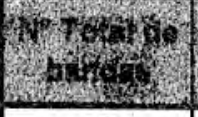 & 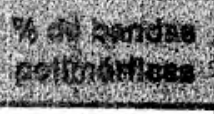 & 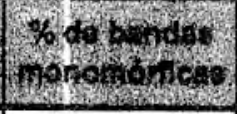 & 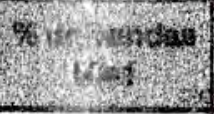 & 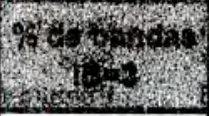 & $\begin{array}{l}\text { o do bundas: } \\
\text { 18w5 }\end{array}$ \\
\hline 1 & AGG/CAC & 26 & 76,92 & 23,08 & 42,31 & 30,77 & 26,92 \\
\hline 2 & ACC/CTG & 33 & 78,79 & 21,21 & 69,69 & 24,24 & 6,07 \\
\hline 3 & ACC/CAT & 43 & 79,06 & 20,94 & 39,53 & 44,19 & 16,28 \\
\hline 4 & ACG/CAC & 28 & 75,00 & 25,00 & 25,00 & 60,71 & 14,29 \\
\hline 5 & AAC/CTC & 29 & 96,55 & 3,45 & 10,34 & 68,97 & 20,69 \\
\hline 6 & AGC/CAG & 23 & 65,22 & 34,78 & 60,87 & 21,74 & 17,39 \\
\hline 7 & $\mathrm{AAC} / \mathrm{CTG}$ & 37 & 86,49 & 13,51 & 0,00 & 62,16 & 37,84 \\
\hline 8 & $\mathrm{ACC} / \mathrm{CTG}$ & 30 & 66,67 & 33,33 & 63,33 & 33,33 & 3,34 \\
\hline \multicolumn{2}{|r|}{ PROMEDIO } & 249 & 78,09 & 21,91 & 38,88 & 43,26 & 17,86 \\
\hline
\end{tabular}

En este cuadro se encuentran los porcertajes de bandas polimórficas y monomórficas obtenidos con cada combinación de primers. Observamos que la combinación de primers AAC/CTC tiene el más alto porcentaje de bandas polimbrificas $(96,55 \%)$ y la combinación AGC/CAG tiene ol más bajo porcentaje de BPM $(65,22 \%)$; y en total, usando las $\propto$ cho combinaciones de primers tenemos un promedio de $78,09 \%$ de BPM y $21,91 \%$ de BMM. Esto quiere decir que un $78,09 \%$ de las bandas totales han aportado para diferenciar los genotipos de camote en estudio 
Figura $\mathbf{N}^{\circ} 01$

Dendograma obtenido de la caracterización molecular de 25 genotipos de camote de Tacna evaluados en el año 2001.

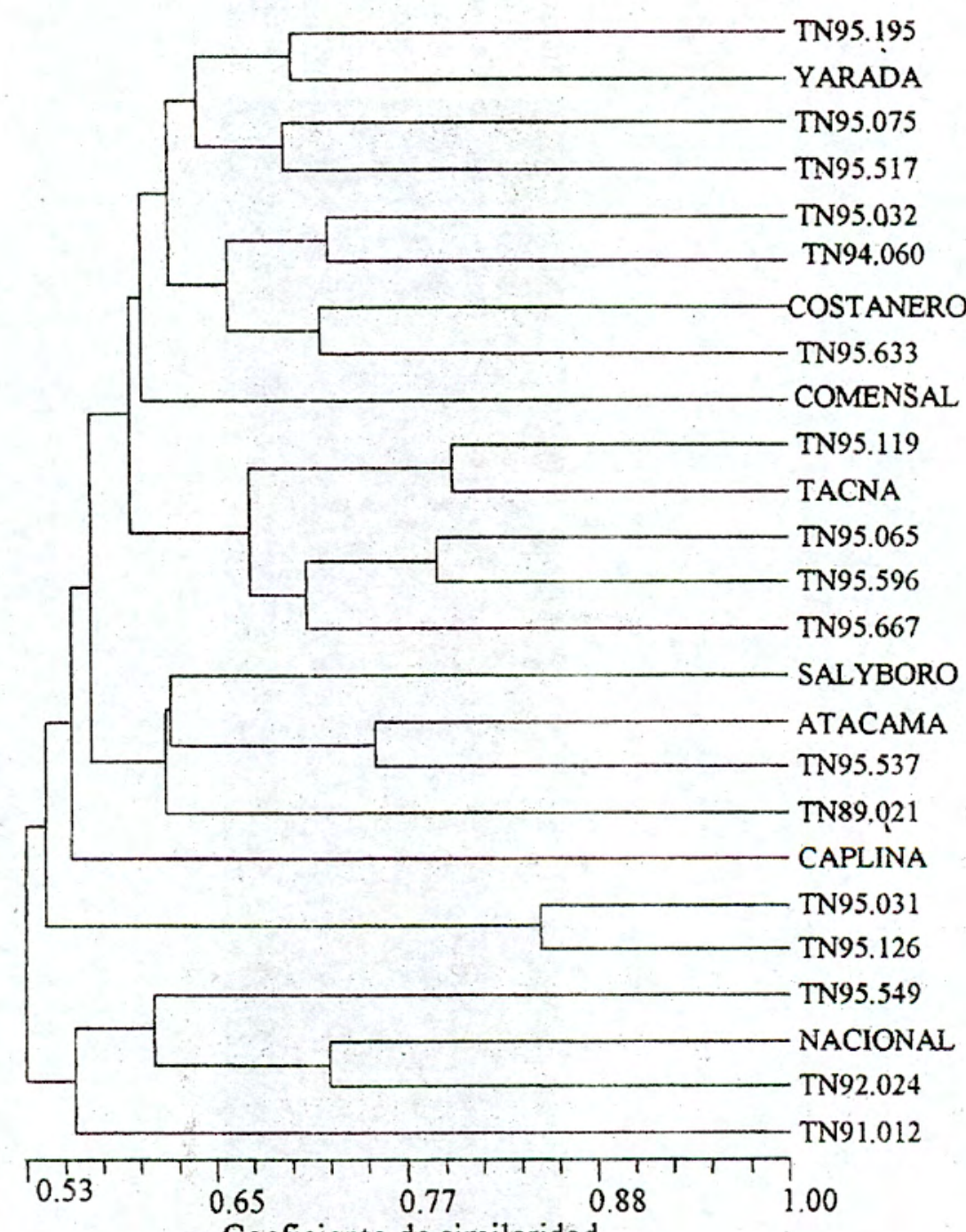

Coeficiente de similaridad 
Figura $\mathrm{N}^{\circ} \mathrm{O2}$

Patrón de bandas electroforéticas obtenidas de la caracterización molecular de 10 genotipos de camote de Tacna. 2004

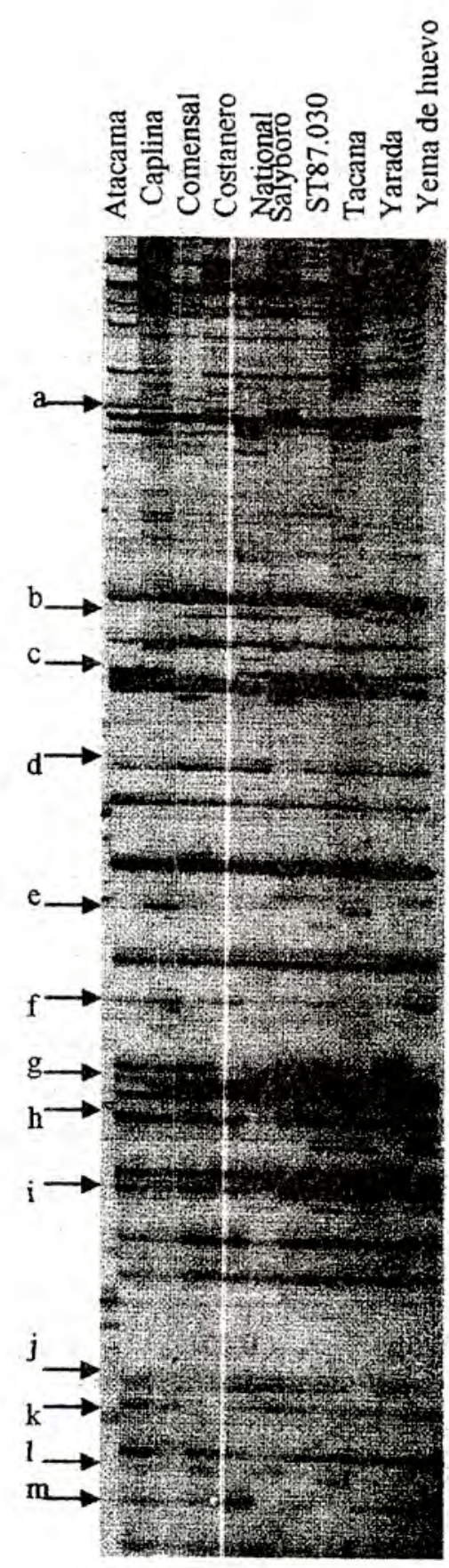


Cuadro $\mathrm{N}^{\circ}$ 05: Descripción de la presencia-ausencia de bandas electroforéticas de las 10 variedades procedentes de Tacna. Tacna. 2004

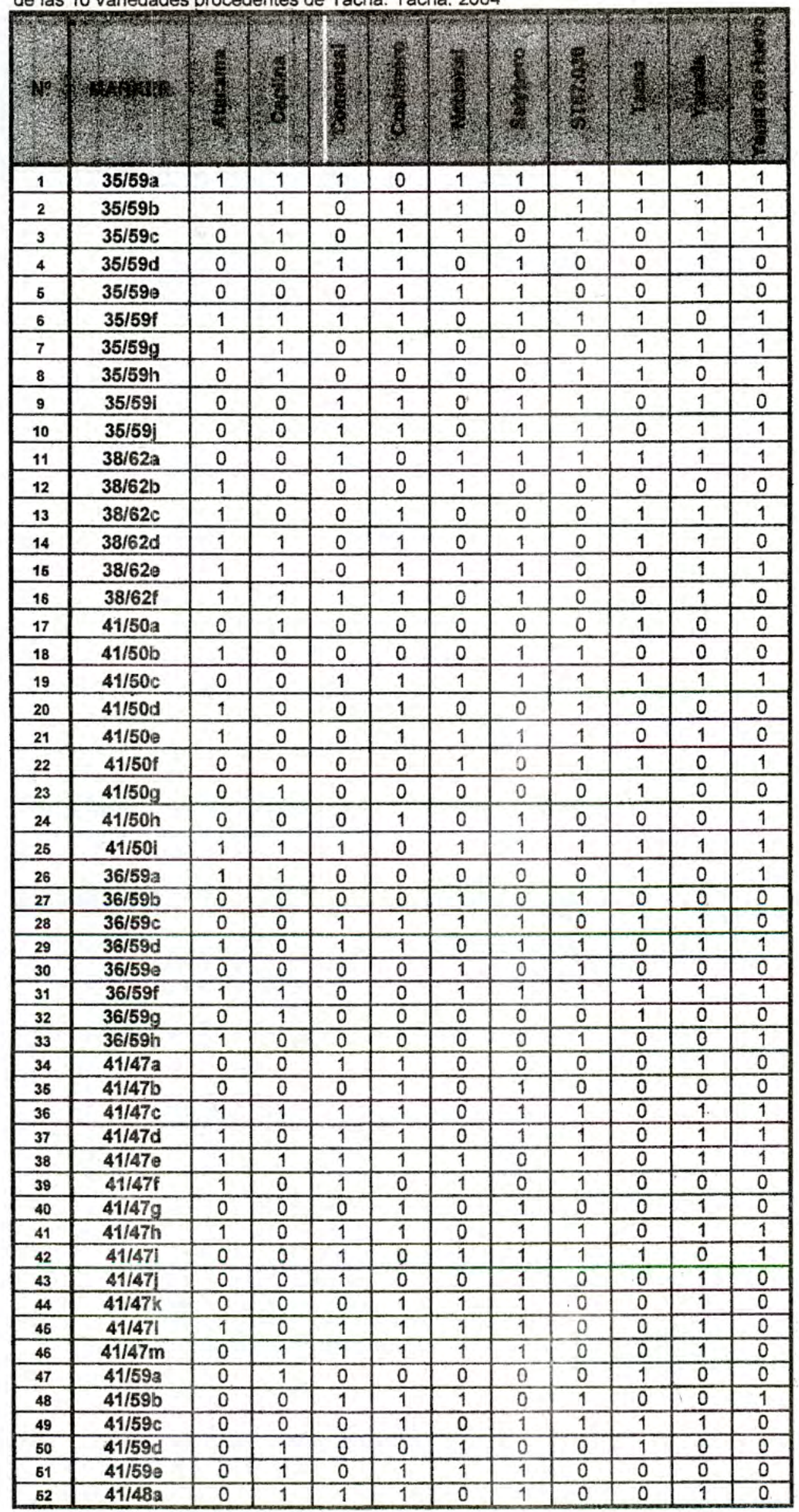




\section{Continuación}

\begin{tabular}{|c|c|c|c|c|c|c|c|c|c|c|c|}
\hline $\mathbf{N}^{0}$ & MARKER & $\frac{g}{8}$ & $\frac{\pi}{\frac{\pi}{8}}$ & $\begin{array}{l}\text { ॠ } \\
\frac{8}{8} \\
\frac{E}{8}\end{array}$ & $\frac{2}{\frac{8}{8}}$ & $\begin{array}{l}\frac{\pi}{6} \\
\frac{0}{8} \\
\frac{10}{2}\end{array}$ & $\frac{\frac{2}{8}}{\frac{8}{6}}$ & $\frac{8}{8}$ & 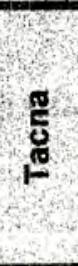 & 용 & 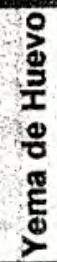 \\
\hline 53 & $41 / 48 b$ & 1 & 1 & 1 & 1 & 1 & 0 & 1 & 1 & 1 & 1 \\
\hline 54 & $41 / 48 \mathrm{c}$ & 0 & 1 & 0 & 0 & 0 & 1 & 0 & 1 & 1 & 0 \\
\hline 55 & $41 / 48 d$ & 0 & 0 & 0 & 1 & 0 & 1 & 0 & 1 & 0 & 0 \\
\hline 56 & $41 / 48 \mathrm{e}$ & 0 & 0 & 0 & 1 & 0 & 1 & 0 & 0 & 1 & 0 \\
\hline 57 & $41 / 48 \mathrm{f}$ & 0 & 0 & 0 & 0 & 1 & 0 & 1 & 0 & 0 & 0 \\
\hline 58 & $41 / 48 \mathrm{~g}$ & 0 & 0 & 1 & 1 & 0 & 1 & 0 & 0 & 0 & 0 \\
\hline 59 & $41 / 48 h$ & 0 & 0 & 1 & 0 & 1 & 0 & 1 & 0 & 0 & 0 \\
\hline 60 & $41 / 48 j$ & 0 & 0 & 0 & 0 & 1 & 1 & 1 & 1 & 1 & 0 \\
\hline 61 & $41 / 48 \mathrm{k}$ & 0 & 1 & 1 & 0 & 1 & 0 & 1 & 1 & 1 & 0 \\
\hline 62 & $41 / 481$ & 0 & 1 & 1 & 0 & 1 & 0 & 1 & 1 & 1 & 1 \\
\hline 63 & $41 / 48 \mathrm{~m}$ & 0 & 1 & 1 & 1 & 1 & 1 & 1 & 0 & 0 & 1 \\
\hline 64 & $41 / 48 n$ & 1 & 1 & 0 & 1 & 0 & 1 & 0 & 1 & 1 & 1 \\
\hline 65 & $41 / 480$ & 1 & 1 & 0 & 1 & 1 & 1 & 0 & 1 & 1 & 0 \\
\hline 66 & $41 / 48 p$ & 1 & 1 & 1 & 1 & 0 & 1 & 0 & 1 & 1 & 1 \\
\hline 67 & $41 / 48 q$ & 0 & 1 & 0 & 1 & 1 & 1 & 1 & 0 & 1 & 0 \\
\hline 68 & $41 / 48 \mathrm{r}$ & 0 & 0 & 0 & 0 & 1 & 0 & 1 & 0 & 1 & 0 \\
\hline 69 & $41 / 48 \mathrm{~s}$ & 0 & 0 & 1 & 1 & 1 & 0 & 0 & 0 & 0 & 0 \\
\hline 70 & $40 / 62 a$ & 1 & 1 & 0 & 1 & 1 & 1 & 1 & 1 & 1 & 1 \\
\hline 71 & $40 / 62 b$ & 1 & 1 & 0 & 1 & 0 & 1 & 0 & 0 & 0 & 0 \\
\hline 72 & $40 / 62 c$ & 0 & 0 & 1 & 1 & 1 & 1 & 0 & 0 & 1 & 0 \\
\hline 73 & $40 / 62 d$ & 1 & 1 & 0 & 0 & 1 & 1 & 1 & 1 & 1 & 1 \\
\hline 74 & $40 / 62 \mathrm{e}$ & 1 & 1 & 1 & 1 & 1 & 0 & 1 & 1 & 1 & 1 \\
\hline 75 & $40 / 62 f$ & 0 & 1 & 0 & 0 & 0 & 0 & 0 & 1 & 0 & 0 \\
\hline 76 & $40 / 62 \mathrm{~g}$ & 1 & 1 & 0 & 1 & 0 & 0 & 1 & 0 & 0 & 1 \\
\hline 77 & $40 / 62 \mathrm{~h}$ & 1 & 1 & 1 & 0 & 0 & 1 & 1 & 0 & 1 & 0 \\
\hline 78 & $40 / 62 i$ & 1 & 1 & 1 & 1 & 0 & 1 & 1 & 1 & 1 & 1 \\
\hline 79 & $40 / 62 j$ & 0 & 0 & 0 & 0 & 0 & 0 & 1 & 1 & 0 & 1 \\
\hline 80 & $40 / 62 k$ & 1 & 1 & 1 & 1 & 0 & 1 & 1 & 1 & 1 & 1 \\
\hline 81 & $40 / 621$ & 1 & 0 & 0 & 0 & 1 & 0 & 1 & 1 & 0 & 1 \\
\hline 82 & $40 / 62 \mathrm{~m}$ & 1 & 0 & 0 & 1 & 1 & 1 & 1 & 0 & 1 & 1 \\
\hline 83 & $40 / 62 n$ & 1 & 1 & 0 & 1 & 1 & 1 & 1 & 1 & 1 & 1 \\
\hline 84 & $40 / 620$ & 1 & 1 & 1 & 1 & 0 & 1 & 0 & 1 & 1 & 1 \\
\hline 85 & $32 / 49 a$ & 0 & 0 & 0 & 0 & 1 & 0 & 1 & 0 & 0 & 0 \\
\hline 86 & $32 / 49 b$ & 1 & 0 & 1 & 1 & 1 & 1 & 1 & 1 & 0 & 0 \\
\hline 87 & $32 / 49 c$ & 1 & 1 & 1 & 0 & 0 & 1 & 0 & 0 & 1 & 0 \\
\hline 88 & $32 / 49 d$ & 0 & 1 & 1 & 1 & 1 & 0 & 1 & 1 & 1 & 1 \\
\hline 89 & $32 / 49 \mathrm{e}$ & 1 & 1 & 1 & 1 & 1 & 0 & 1 & 1 & 1 & 1 \\
\hline 90 & $32 / 49 \mathrm{f}$ & 1 & 1 & 1 & 1 & 0 & 1 & 0 & 1 & 1 & 0 \\
\hline 91 & $32 / 49 \mathrm{~g}$ & 0 & 0 & 0 & 1 & 1 & 0 & 1 & 0 & 0 & 0 \\
\hline 92 & $32 / 49 \mathrm{~h}$ & 1 & 1 & 0 & 1 & 0 & 0 & 1 & 1 & 1 & 0 \\
\hline 93 & $32 / 49 i$ & 1 & 1 & 1 & 1 & 1 & 1 & 0 & 1 & 1 & 1 \\
\hline 94 & $32 / 49 j$ & 1 & 0 & 0 & 0 & 0 & 0 & 1 & 1 & 0 & 1 \\
\hline 95 & $32 / 49 \mathrm{k}$ & 1 & 1 & 0 & 0 & 1 & 1 & 1 & 1 & 1 & 1 \\
\hline 96 & $32 / 491$ & 1 & 1 & 0 & 0 & 0 & 0 & 0 & 0 & 0 & 1 \\
\hline 97 & $32 / 49 \mathrm{~m}$ & 0 & 0 & 1 & 1 & 1 & 1 & 1 & 0 & 1 & 0 \\
\hline 98 & $32 / 49 n$ & 0 & 1 & 0 & 0 & 1 & 0 & 0 & 1 & 0 & 1 \\
\hline
\end{tabular}


CUADRO $N^{\circ}$ 06: Número y porcentaje de marcadores polimórficos presentes por cada genotipo en estudio, de un total de 98 marcadores evaluados

\begin{tabular}{|c|l|c|c|}
\hline $\mathbf{N}^{\circ}$ & \multicolumn{1}{|c|}{ Genotipo } & $\begin{array}{c}\text { Número de } \\
\text { marcadores } \\
\text { presentes }\end{array}$ & $\begin{array}{c}\text { Porcentaje } \\
\text { (\%) }\end{array}$ \\
\hline $\mathbf{1}$ & Atacama & 48 & 48,98 \\
$\mathbf{2}$ & Caplina & 51 & 52,04 \\
$\mathbf{3}$ & Comensal & 44 & 44,90 \\
$\mathbf{4}$ & Costanero & 60 & 61,22 \\
$\mathbf{5}$ & Nacional & 51 & 52,04 \\
$\mathbf{6}$ & Salyboro & 57 & 58,16 \\
$\mathbf{7}$ & ST87.030 & 56 & 57,14 \\
$\mathbf{8}$ & Tacna & 49 & 50,00 \\
$\mathbf{9}$ & Yarada & 60 & 61,22 \\
$\mathbf{1 0}$ & Yema de Huevo & 47 & 47,96 \\
\hline
\end{tabular}

Figura $\mathrm{N}^{\circ} 03$

Dendograma obtenido de la caraterización molecular de los 10 genotipos de camote de Tacna evaluados en el aก๊o 2004.

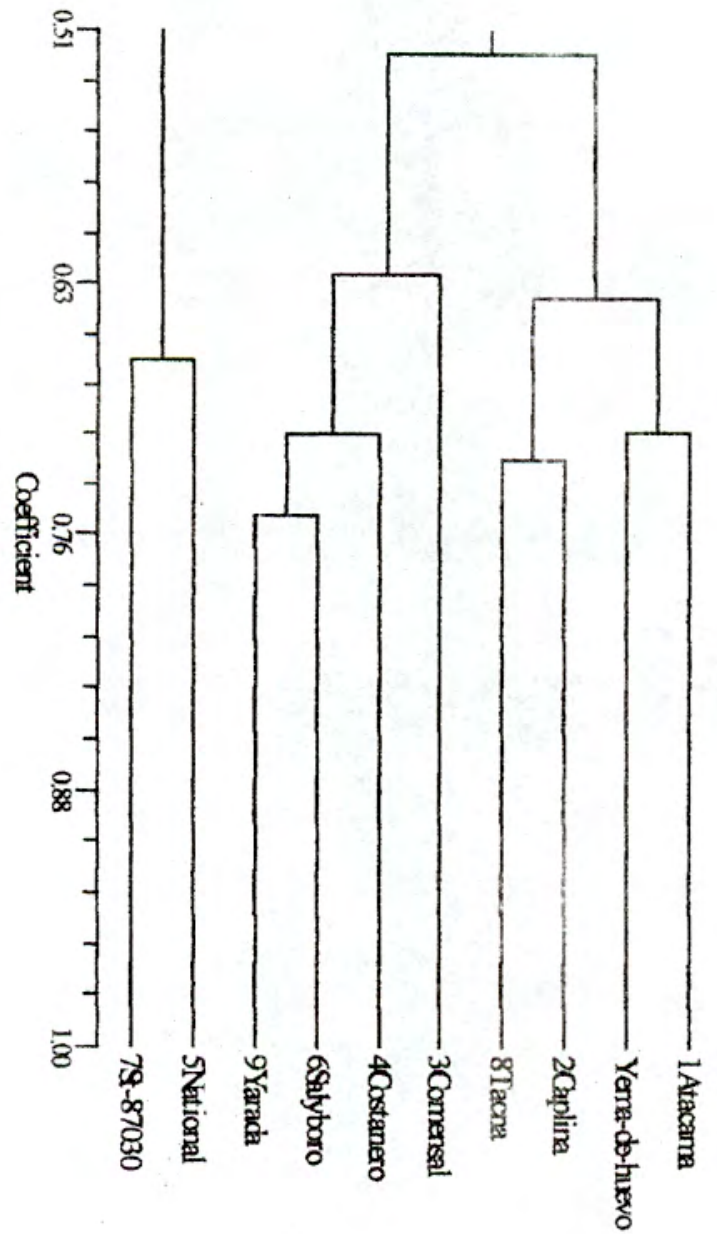




\section{Figura $\mathrm{N}^{\circ} 04$}

Patrón de bandas electroforéticas obtenidas de la caracterización molecular de 19 genotipos de camote de Tacna, evaluados en Diciembre del 2004.

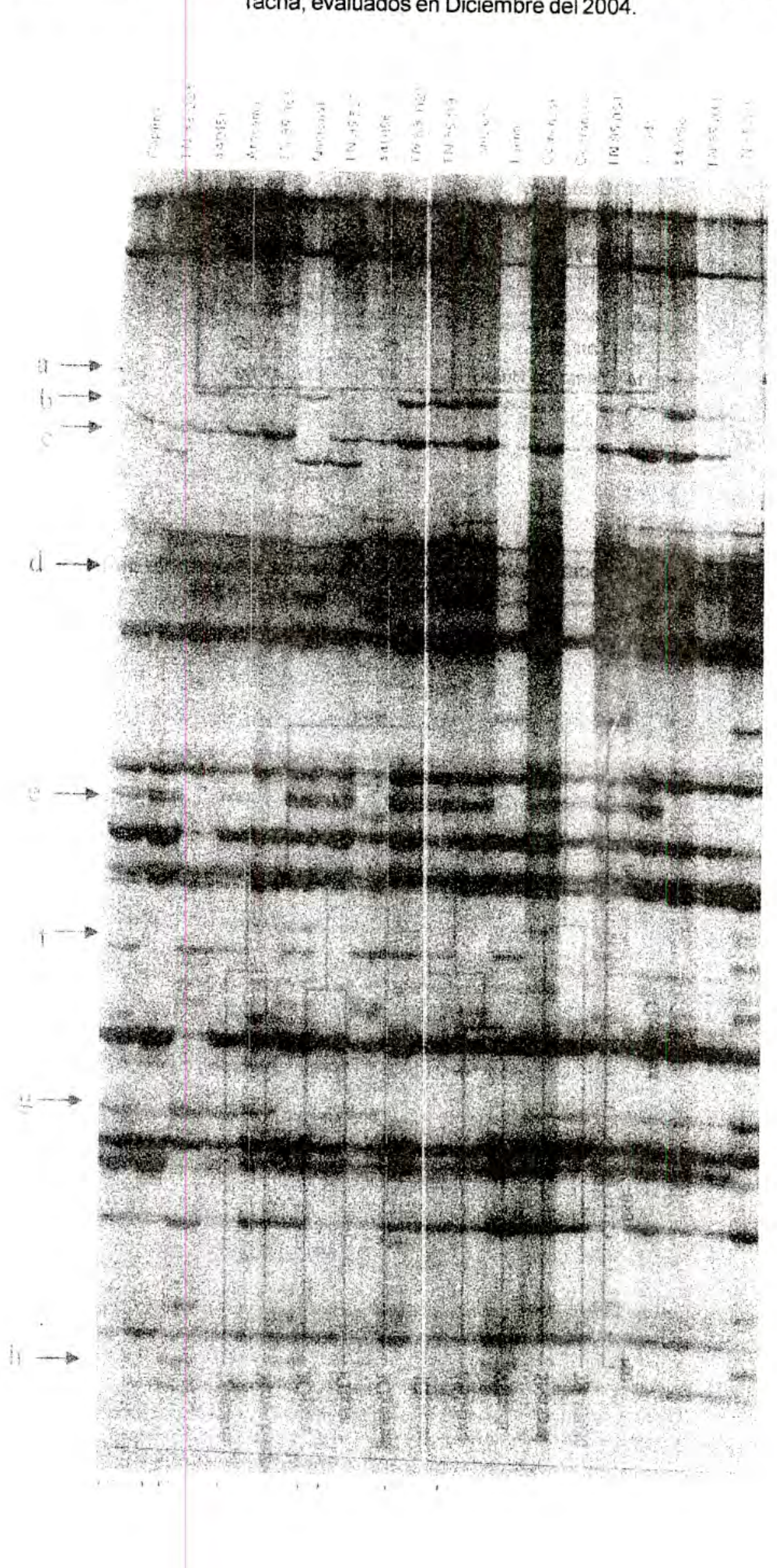


CUADRO $N^{\circ}$ 07: Descripción de la presencia - ausencia de bandas electroforéticas de los 19 genotipos evaluados. Tacna, 2004

\begin{tabular}{|c|c|c|c|c|c|c|c|c|c|c|c|c|c|c|c|c|c|c|c|c|}
\hline $\mathrm{N}^{0}$ & MARKER & 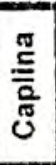 & 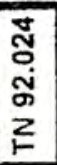 & $\frac{5}{\frac{\pi}{8}}$ & 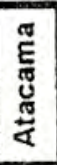 & $\begin{array}{l}0 \\
0 \\
0 \\
\dot{0} \\
z \\
1\end{array}$ & $\begin{array}{l}\text { ल] } \\
\frac{\pi}{0} \\
\frac{0}{0} \\
\frac{\pi}{2}\end{array}$ & 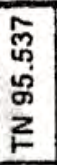 & $\frac{\mathscr{0}}{\frac{0}{0}}$ & $\begin{array}{l}\overline{\text { s }} \\
\dot{\Phi} \\
\Phi \\
z\end{array}$ & $\begin{array}{l}\frac{0}{2} \\
\text { \& } \\
2 \\
2\end{array}$ & 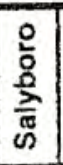 & 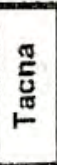 & 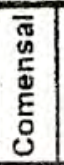 & 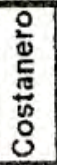 & 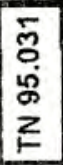 & 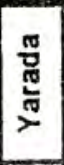 & $\frac{\infty}{0}$ & $\begin{array}{l}5 \\
0 \\
\dot{\infty} \\
z \\
z\end{array}$ & $\begin{array}{l}n \\
5 \\
0 \\
\infty \\
2 \\
2 \\
r\end{array}$ \\
\hline 1 & $2 \mathrm{M} 60 \mathrm{a}$ & 0 & 0 & 0 & 0 & 1 & 1 & 1 & 1 & 0 & 0 & 0 & 1 & 0 & 1 & 1 & 1 & 0 & 1 & - \\
\hline 2 & E32M60b & 0 & 1 & 0 & 0 & 1 & 1 & 1 & 0 & 1 & 1 & 0 & 1 & 1 & 1 & 1 & 1 & & 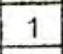 & 0 \\
\hline 3 & E32M60c & 0 & 0 & 1 & 0 & 1 & 1 & 1 & 1 & 1 & 1 & 1 & 1 & 0 & 1 & 1 & 0 & 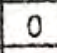 & 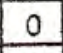 & 1 \\
\hline 4 & $2 \mathrm{MGOd}$ & 0 & 0 & 0 & 0 & 0 & 0 & 0 & 1 & 0 & 0 & 0 & 0 & 0 & 0 & 0 & 0 & 0 & 0 & 0 \\
\hline 5 & ge & 1 & 1 & 19 & 1 & 0 & 1 & -1 & 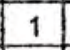 & 1 & - & 1 & 0 & 1 & 1 & 1 & 1 & 1 & 1 & 1 \\
\hline 6 & A60f & 1 & 0 & 0 & 1 & 0 & 0 & - & 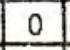 & . & 0 & 0 & 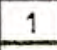 & 1 & 1 & 1 & 1 & 0 & 0 & 1 \\
\hline 7 & $60 \mathrm{~g}$ & 0 & 0 & 0 & 0 & 0 & 0 & 0 & 1 & 0 & 0 & 0 & 0 & -7 & 0 & 0 & 0 & 0 & 0 & 0 \\
\hline 8 & $\mathrm{Oh}$ & 1 & 1 & 0 & 1 & 0 & 1 & 1 & 0 & 1 & 0 & 0 & $-1+3$ & 0 & 0 & 0 & 1 & 1 & 1 & 0 \\
\hline 9 & E32M60i & 0 & 0 & 1 & 0 & 0 & 1 & 0 & 1 & 0 & 0 & 1 & 1 & 1 & 1 & 1 & 1 & 1 & 1 & 1 \\
\hline 10 & $80 \mathrm{j}$ & 0 & 0 & 0 & 0 & 1 & 0 & 0 & 0 & 0 & 0 & 1 & 0 & 1 & 1 & 1 & 1 & 1 & 1 & 0 \\
\hline 11 & $k$ & 1 & 1 & 0 & 1 & 1 & 1 & 1 & 0 & 1 & 1 & 1 & 1 & 1 & 1 & 1 & 1 & 1 & 1 & 0 \\
\hline 12 & E. & 1 & 0 & 1 & 1 & 0 & 1 & 0 & $=$ & 0 & 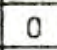 & 0 & 0 & 0 & 0 & 1 & 1 & 1 & 1 & 1 \\
\hline 13 & E3 & 1 & 1 & 0 & 0 & 1 & 1 & 1 & 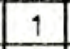 & 1 & 1 & 1 & 1 & 1 & $r_{1}$ & 0 & 1 & 1 & 1 & 1 \\
\hline 14 & $0 n$ & 1 & 0 & 0 & 0 & 1 & 1 & 0 & 1 & 1 & 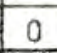 & 0 & 1 & 0 & 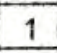 & 1 & 1 & 1 & 1 & 0 \\
\hline 15 & 00 & 0 & 0 & 1 & 0 & 1 & 0 & 0 & 1 & 0 & 1 & 1 & 1 & 1 & 1 & 1 & 1 & 0 & 0 & 1 \\
\hline 16 & $60 p$ & 1 & 1 & 0 & 1 & 1 & 1 & 1 & 1 & 0 & 1 & 0 & 1 & 1 & 1 & 1 & 1 & 1 & 1 & 1 \\
\hline 17 & $30 q$ & 0 & 0 & 1 & 0 & 0 & 0 & 0 & 0 & 0 & 0 & 1 & 0 & 0 & 0 & 1 & 0 & 0 & 0 & 0 \\
\hline 18 & $2 a$ & 0 & 1 & 0 & 0 & 1 & 1 & 1 & 0 & 1 & 0 & 1 & 1 & 0 & 1 & 0 & 1 & 1 & 1 & 0 \\
\hline 19 & & 1 & 1 & 1 & 1 & 1 & 1 & 1 & 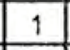 & 1 & 1 & 0 & 1 & 0 & 1 & 1 & 1 & 1 & 1 & 1 \\
\hline 20 & E3 & 1 & 1 & 0 & 1 & 1 & 1 & 1 & 1 & 1 & 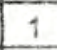 & 0 & 1 & 1 & 1 & 1 & 1 & 1 & 1 & 1 \\
\hline 21 & $2 d$ & 1 & 1 & 1 & 1 & 1 & 1 & 1 & 1 & 1 & - & 1 & 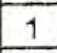 & 1 & 1 & 0 & 1 & 1 & 1 & 1 \\
\hline 22 & $2 e$ & 0 & 0 & 1 & 0 & 0 & 1 & 0 & 0 & 0 & 0 & 0 & 0 & 1 & 0 & 1 & 1 & 0 & 0 & 0 \\
\hline 23 & $2 f$ & 0 & 0 & 0 & 0 & 1 & 1 & 0 & 0 & 0 & 0 & 0 & 0 & 0 & 0 & 0 & 0 & 0 & 0 & 0 \\
\hline 24 & $32 \mathrm{~g}$ & 1 & 1 & 0 & 1 & 1 & 1 & 1 & 1 & 1 & 1 & 1 & 1 & 1 & 1 & 1 & 1 & 1 & 1 & 1 \\
\hline 25 & $2 \mathrm{~h}$ & 0 & 0 & 0 & 0 & 1 & 0 & 0 & 0 & 0 & 0 & 0 & 0 & 0 & 1 & 1 & 1 & 0 & 0 & 0 \\
\hline 25 & E & 0 & 0 & 0 & 0 & 1 & 1 & 0 & 0 & 1 & 1 & 0 & 1 & 1 & 0 & 1 & 1 & 0 & 0 & 0 \\
\hline 27 & E & 1 & 1 & - & 1 & - & 1 & 1 & - & 1 & + & 1 & 1 & 1 & 1 & 0 & 1 & 1 & 1 & 1 \\
\hline 28 & E32MB2k & 1 & 0 & 0 & 1 & 1 & 1 & 0 & 0 & 0 & 1 & 0 & 0 & 0 & 0 & 0 & 0 & 0 & 0 & 0 \\
\hline 29 & a & 0 & 0 & 1 & 0 & 0 & 0 & 0 & 0 & 0 & 0 & 0 & 1 & 0 & 0 & 0 & 0 & 0 & 0 & 0 \\
\hline 30 & bb & 0 & 0 & 0 & 0 & 0 & 1 & 0 & 1 & 1 & 0 & 0 & 0 & $c$ & 0 & 0 & 0 & 1 & 1 & 1 \\
\hline 31 & $9 c$ & 9 & 1 & 0 & 1 & 0 & 0 & 1 & 1 & 1 & 1 & 0 & 0 & 1 & 1 & 0 & 1 & 0 & 0 & 0 \\
\hline 32 & E44M39d & 1 & 1 & 0 & 1 & 1 & 1 & 1 & 1 & 1 & 1 & 1 & 1 & 1 & 1 & 1 & 1 & 1 & 1 & 1 \\
\hline 33 & E & 0 & 0 & 0 & 0 & 1 & 0 & 0 & 0 & 0 & 0 & 0 & 0 & 1 & 0 & 0 & 0 & 0 & 0 & 0 \\
\hline 34 & E44M39f & 1 & 1 & 0 & 1 & $x_{10}$ & 1 & 1 & 1 & 1 & 0 & 1 & 1 & C & 0 & 1 & 1 & 0 & 0 & 0 \\
\hline 35 & E & 0 & 0 & 0 & 0 & 0 & 0 & 0 & 1 & 0 & 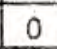 & 0 & 0 & 0 & 0 & 0 & 0 & 0 & 0 & 1 \\
\hline 36 & $9 \mathrm{~h}$ & 0 & 0 & 1 & 1 & 1 & 0 & 0 & 1 & 0 & 0 & 1 & 0 & 1 & 1 & 1. & 1 & 0 & 0 & 0 \\
\hline 37 & E44M39i & 1 & 0 & 0 & 1 & 1 & 1 & 0 & 1 & 1 & 0 & 1 & 0 & $C$ & 0 & 1 & 1 & 1 & 1 & 0 \\
\hline 38 & E44M39j & 0 & 1 & 1 & 1 & 1 & 1 & 1 & 1 & 1 & 1 & 1 & 0 & 1 & 1 & 0 & 1 & 0 & 0 & 1 \\
\hline 39 & E44 & 0 & 1 & 0 & 0 & 0 & 0 & 1 & 0 & 1 & 0 & 0 & 0 & 1 & 0 & 0 & 0 & 0 & 0 & 0 \\
\hline 40 & $E$ & 0 & 0 & 1 & 0 & 0 & 0 & 0 & 0 & 0 & 0 & 0 & 1 & 0 & 0 & 0 & 0 & 0 & 0 & 0 \\
\hline 41 & E36M61a & 1 & 0 & 1 & 1 & 1 & 1 & 0 & 0 & 1 & 1 & $i$ & 1 & 1 & 1 & 1 & 1 & 1 & 1 & 1 \\
\hline 42 & $61 \mathrm{~b}$ & 1 & 1 & 1 & 1 & 1 & 0 & 1 & 1 & 1 & 1 & 1 & 1 & 1 & 1 & 1 & 1 & 1 & 1 & 0 \\
\hline 43 & E36M61c & 1 & 1 & 0 & 0 & 0 & 1 & 1 & 0 & 0 & 0 & 0 & 0 & 0 & 0 & 0 & 0 & 0 & 0 & 0 \\
\hline 44 & E36M51d & 0 & 0 & 0 & 0 & 0 & 0 & 0 & 1 & 0 & 0 & 0 & 1 & 0 & 0 & 1 & 0 & 0 & 0 & 1 \\
\hline 45 & $E$ & 7 & 1 & 0 & 1 & 0 & 1 & 1 & 0 & 1 & 1 & 1 & 0 & 1 & 1 & 1 & 1 & 0 & 0 & 0 \\
\hline 46 & ES6OVIVTt & 0 & 0 & 0 & 0 & 0 & 0 & 0 & 1 & 0 & 0 & 0 & 0 & 0 & 0 & 0 & 0 & 0 & 0 & 0 \\
\hline 47 & E36M61g & 1 & 0 & 1 & 1 & 0 & 1 & 0 & 1 & 1 & 1 & 0 & 1 & 0 & 0 & 1 & 0 & 0 & 0 & 1 \\
\hline 48 & E36M61h & 0 & 0 & 1 & 0 & 0 & 1 & 0 & 1 & 0 & 0 & 1 & 0 & 0 & 0 & 0 & 0 & 1 & 0 & 1 \\
\hline 49 & M61i & 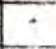 & 1 & 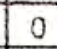 & 1 & $i$ & 0 & 1 & 0 & 0 & (1) & 1 & 1 & 0 & 0 & 0 & 0 & 0 & 0 & 2 \\
\hline 50 & E36M61j & & 1 & 1 & 1 & 1 & U & 1 & 11 & 0 & 0 & & 0 & & 1 & & 1 & 1 & 1 & \\
\hline
\end{tabular}




\begin{tabular}{|c|c|c|c|c|c|c|c|c|c|c|c|c|c|c|c|c|c|c|c|c|}
\hline $\mathrm{N}^{\circ}$ & MARKER & 祢 & 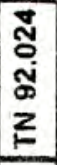 & $\frac{5}{5}$ & 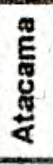 & $\begin{array}{l}\mathscr{8} \\
8 \\
10 \\
2 \\
z\end{array}$ & 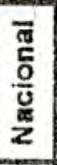 & 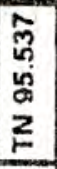 & 㔛 & \begin{tabular}{|c}
$\bar{y}$ \\
0 \\
$\dot{\infty}$ \\
$z$ \\
\end{tabular} & $\begin{array}{l}0 \\
\frac{9}{2} \\
5 \\
z \\
z\end{array}$ & $\frac{8}{8}$ & 总 & 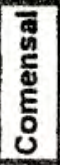 & 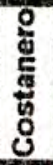 & $\begin{array}{l}-5 \\
\text { o. } \\
\text { \&े } \\
z\end{array}$ & $\begin{array}{l}\frac{\pi}{\tilde{w}} \\
\frac{\sqrt{5}}{2} \\
\nu\end{array}$ & $\frac{\pi}{\frac{16}{9}}$ & $\begin{array}{c}\bar{\sigma} \\
0 \\
\dot{\alpha} \\
z \\
-\end{array}$ & 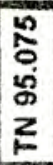 \\
\hline 51 & E36M61k & 0 & 0 & 1 & 0 & 0 & 1 & 0 & & 0 & 0 & 0 & 1 & 0 & 0 & 0 & 0 & 0 & 0 & 1 \\
\hline 62 & E38M611 & 1 & 1 & 1 & 1 & 1 & 1 & 1 & 0 & 1 & 1 & 1 & 0 & 1 & 0 & 0 & 1 & 1 & 1 & 0 \\
\hline 53 & E46M36a & 1 & 1 & 0 & 0 & 1 & 0 & 1 & 1 & 1 & 0 & 0 & 0 & 0 & 0 & 0 & 0 & 0 & 0 & 0 \\
\hline 54 & $\mathrm{E} 46 \mathrm{M} 36 \mathrm{~b}$ & 0 & 0 & 0 & 1 & 0 & 0 & 0 & 0 & 0 & 0 & 0. & 0 & 0 & 1 & 0 & 0 & 0 & 0 & 0 \\
\hline 55 & $\mathrm{E} 46 \mathrm{M} 36 \mathrm{C}$ & 0 & 0 & 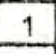 & 1 & 0 & 0 & 0 & 0 & 0 & 0 & 1 & 0 & 0 & 0 & 0 & 0 & 0 & 0 & 0 \\
\hline 56 & E46: & 0 & 0 & 1 & 0 & 1 & 0 & $C$ & 1 & 0 & 0 & 0 & 0 & 1 & 1 & 0 & 1 & 1 & 1 & 1 \\
\hline 57 & E46M36e & 0 & 0 & 0 & 0 & 0 & 1 & 0 & . & 1 & 1 & 0 & 1 & 1 & 0 & 0 & 0 & 0 & 0 & 1 \\
\hline 58 & E46M38f & 1 & 0 & 0 & 1 & 1 & 1 & 0 & 1 & 0 & 1 & 1 & 1 & 1 & 1 & 1 & 0 & 0 & 0 & 1 \\
\hline 59 & $E 46 \mathrm{M} 36 \mathrm{~g}$ & 0 & 0 & 1 & 0 & 0 & 1 & 0 & 1 & 0 & 1 & 1 & 1 & 1 & 1 & 0 & 0 & 0 & 0 & 0 \\
\hline 60 & E46M36h & 1 & 1 & 0 & 1 & 1 & 0 & 1 & 1 & 0 & 1 & 1 & 1 & 1 & 0 & 1 & 0 & 0 & 0 & 1 \\
\hline 61 & E35M42a & 0 & 0 & 0 & 0 & 1 & 1 & 0 & 1 & 0 & 0 & 0 & 0 & 0 & 0 & 1 & 0 & 1 & 1 & 0 \\
\hline 62 & E35M42b & 1 & 1 & 1 & 1 & 1 & 1 & 1 & 1 & 1 & 1 & 0 & 0 & 0 & 0 & 0 & 1 & 0 & 0 & 1 \\
\hline 63 & E35M42c & 1 & 1 & 0 & 1 & 70 & 1 & 1 & 0 & 0 & 0 & 0 & 0 & 0 & 0 & 0 & 0 & 0 & 0 & 1 \\
\hline 64 & E.35! & 1 & 1 & 1 & 1 & 9 & 1 & 1 & 1 & 1 & 1 & 1 & 0 & 1 & 1 & 1 & 0 & 1 & 1 & 1 \\
\hline 65 & E35 & 1 & 0 & 0 & 1 & 1 & 0 & $C$ & 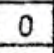 & 1 & 0 & 1 & 1 & 0 & 1 & 0 & 1 & 1 & 1 & 0 \\
\hline 66 & E35 & 0 & 0 & 1 & 0 & 0 & 0 & $c$ & 0 & D & 0 & 1 & 0 & 0 & 0 & 0. & 0 & 0 & 0 & 0 \\
\hline 67 & E35 & 0 & 1 & 0 & 0 & 1 & 1 & 1 & 0 & 0 & 0 & 1 & 0 & 1 & 1 & 0 & 1 & 0 & 0 & 0 \\
\hline 68 & E35M42h & 1 & 0 & 0 & 1 & 0 & 1 & 0 & 0 & 1 & 0 & 1 & 1 & 0 & 1 & 1 & 1 & 1 & 1 & 0 \\
\hline 69 & E35M42i & 1 & 1 & 1 & 1 & 0 & 1 & 1 & 1 & 1 & 1 & 1 & 1 & 1 & 1 & 1 & 1 & 1 & 1 & 1 \\
\hline 70 & E35M42j & 0 & 0 & 0 & 0 & 0 & 0 & 0 & 1 & 0 & 0 & 0 & 0 & 0 & 0 & 0 & 0 & 0 & 0 & 0 \\
\hline 71 & E35M42k & 1 & 1 & 0 & 0 & 0 & 1 & 1 & 0 & 1 & 1 & 1 & 1 & 1 & 1 & 0 & 0 & 0 & 0 & 0 \\
\hline 72 & E35M421 & 0 & 1 & 0 & 0 & 0 & 0 & 1 & 0 & 1 & 1 & 1 & 0 & 0 & 1 & 1 & 1 & 1 & 1 & 1 \\
\hline 73 & $\mathrm{E} 35 \mathrm{M} 42 \mathrm{~m}$ & 0 & 0 & 1 & 0 & 1 & 1 & 0 & 0 & 0 & 0 & 1 & 0 & 0 & 0 & 1 & 0 & 0 & 0 & 0 \\
\hline 74 & E35M42n & 1 & 1 & 0 & 1 & 1 & 1 & 1 & 1 & 1 & 1 & 1 & 1 & 0 & 1 & 1 & 1 & 1 & 1 & 0 \\
\hline 75 & E35M420 & 0 & 0 & 0 & 1 & 1 & 0 & 0 & 0 & 0 & 1 & 1 & 0 & 1 & 1 & 1 & 1 & 1 & 1 & 0 \\
\hline 76 & E35M42p & 1 & 0 & 1 & 1 & 1 & 1 & 0 & 1 & 1 & 1 & 1 & 1 & 1 & 1 & 1 & 1 & 1 & 1 & 0 \\
\hline 77 & E35M42q & 0 & 0 & 0 & 0 & 0 & 0 & 0 & 1 & 0 & 0 & 0 & 0 & 0 & 0 & 0 & 0 & 0 & 0 & 0 \\
\hline 78 & E35M42r & 0 & 0 & 0 & 0 & 0 & 0 & 0 & 1 & 0 & 0 & 0 & 0 & 0 & 0 & 0 & 0 & 0 & 0 & 0 \\
\hline 79 & E35M42s & 1 & 1 & 0 & 1 & 0 & 1 & 1 & 0 & 0 & 0 & 0 & 0 & 0 & 0 & 0 & 0 & 0 & 0 & 1 \\
\hline 80 & E37M60a & 1 & 1 & 0 & 1 & 1 & 1 & 1 & 1 & 1 & 1 & 1 & 0 & 1 & 1 & 0 & 0 & 1 & 1 & 1 \\
\hline 81 & E37M60b & 0 & 0 & 1 & 0 & 0 & 0 & 0 & 1 & 0 & 0 & 0 & 0 & 0 & 0 & 1 & 0 & 0 & 0 & 0 \\
\hline 82 & E37M60c & 1 & 1 & 0 & 1 & 1 & 0 & 1 & 0 & 0 & 0 & 0 & 0 & 1 & 0 & 0 & 1 & 0 & 0 & 0 \\
\hline 83 & E37M80d & 1 & 1 & 0 & 1 & 1 & 1 & 1 & 1 & 1 & 1 & 1 & 1 & 1 & 1 & 1 & 1 & 1 & 1 & 1 \\
\hline 84 & E37M60e & 1 & 1 & 1 & 1 & 1 & 1 & $1]$ & 1 & 1 & 1 & 1 & 0 & 1 & 1 & 0 & 1 & 1 & 1 & 1 \\
\hline 85 & E37M60f & 0 & 0 & 1 & 0 & 0 & 0 & 0 & 0 & 0 & 1 & 0 & 1 & 0 & 0 & 0 & 1 & 0 & 0 & 0 \\
\hline 86 & $E 37 M 60 \mathrm{~g}$ & 0 & 1 & 1 & 0 & 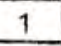 & 0 & 1 & 0 & 1 & 1 & 0 & 0 & 0 & 1 & 0 & 1 & 1 & 1 & 1 \\
\hline 87 & E37M60h & 0 & 1 & 1 & 0 & 0 & 0 & 1 & 1 & 1 & 0 & 0 & 1 & 0 & 0 & 0 & 0 & 0 & 0 & 0 \\
\hline 88 & E37M60i & 0 & 0 & 1 & 0 & 0 & 1 & 0 & 1 & 1 & 0 & 1 & 1 & 0 & 1 & 1 & 1 & 1 & 1 & 0 \\
\hline 89 & E37M60j & 1 & 1 & 0 & 1 & 0 & 1 & 1 & 0 & 0 & 0 & 1 & 1 & 1 & 1 & 1 & 1 & 0 & 0 & 0 \\
\hline 90 & E37M60k & 1 & 0 & 1 & 1 & 0 & 1 & 0 & 1 & 1 & 1 & 1 & 1 & 1 & 1 & 1 & 0 & 1 & 1 & 0 \\
\hline 91 & E37M60I & 1 & 1 & 1 & 1 & 1 & 0 & 1 & 1 & 1 & 1 & 1. & 1 & 1 & 1 & 1 & 1 & 1 & 1 & 1 \\
\hline 92 & E37M60m & 0 & 0 & 1 & 0 & 1 & 1 & 1 & 1 & 1 & 0 & 0 & 1 & 0 & 0 & 0 & 0 & 1 & 1 & 1 \\
\hline 93 & E37MBOn & 0 & 0 & 0 & 0 & 0 & 1 & 0 & 0 & 0 & 0 & 0 & $0]$ & 0 & 0 & 1. & 1 & 1 & 1 & 0 \\
\hline 94 & E37M600 & 0 & 0 & 1 & 0 & 0 & 0 & 0 & 0 & 1 & 0 & 1. & 0 & 1 & 1 & 1 & 1 & 1 & 1 & 1 \\
\hline 96 & E37M80p & 1 & 0 & 1 & 0 & 1 & 1 & 0 & 1 & 0 & 1 & 1 & 1 & 1 & 1 & 1 & 1 & 0 & 0 & 1 \\
\hline 96 & $\mathrm{E} 37 \mathrm{M} 60 \mathrm{q}$ & 0 & 0 & 0 & 0 & 0 & 0 & 0 & 0 & 0 & 0 & 1 & 1 & 0 & 0 & 1 & 0 & 0 & 0 & 1 \\
\hline 97 & E37M60r & 0 & 1 & 0 & 0 & 1 & 1 & 1 & 1 & 1 & 0 & 1 & 1 & 1 & 1 & 1 & 1 & 1 & 1 & 1 \\
\hline 98 & E37M60s & 1 & 0 & 0 & 0 & 1 & 1 & 1 & 0 & 1 & 1 & 1 & 1 & 0 & 1 & 1 & 1 & 1 & 1 & 0 \\
\hline
\end{tabular}


Figura $\mathrm{N}^{\circ} 05$

Dendograma obtenido de la caraterización molecular de los 19 genotipos de camote de Tacna evaluados en Diciembre del 2004.

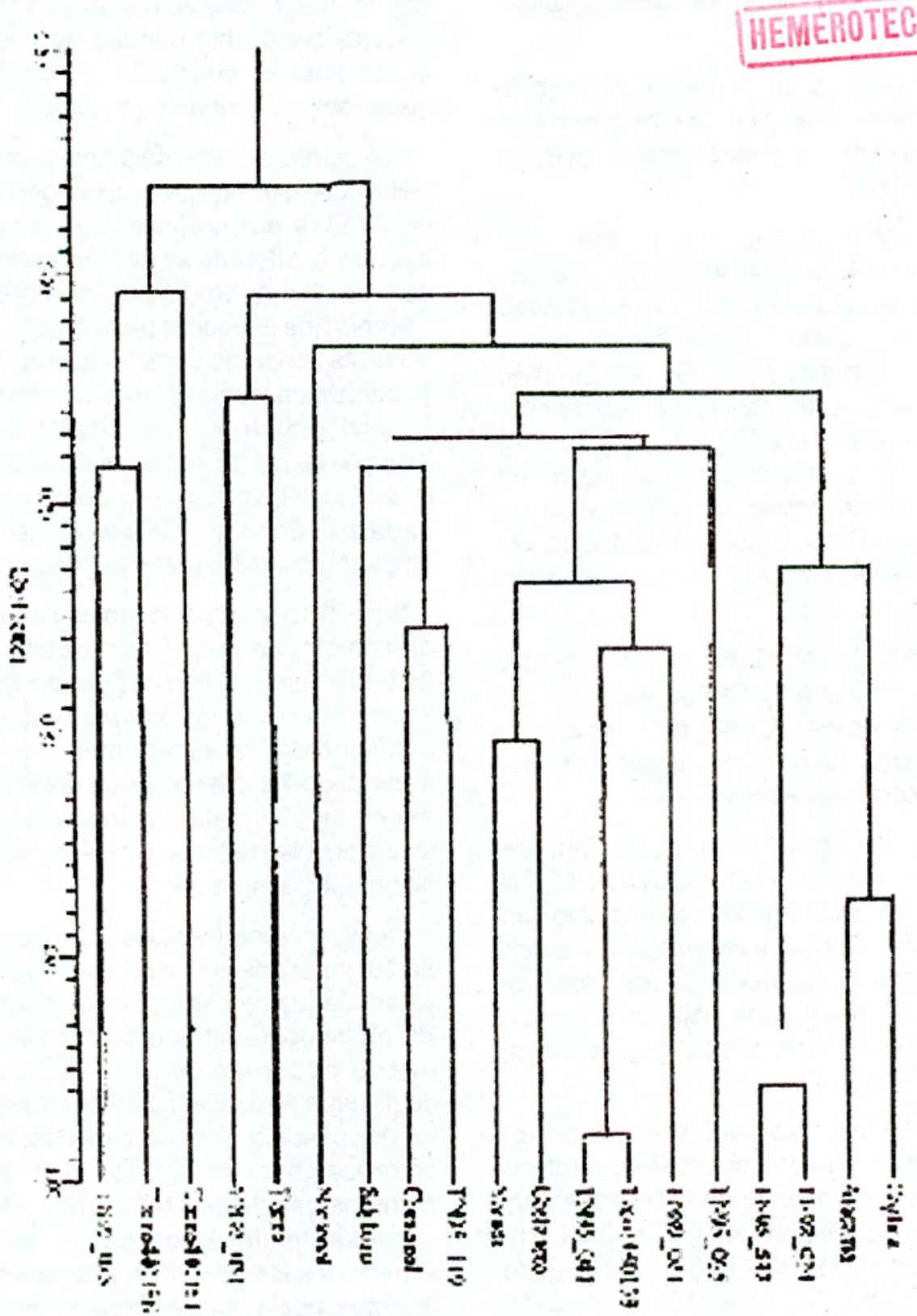




\section{DISCUSIÓN}

Los recursos genéticos de camote corren un riesgo de pérdida en cantidades significativas de germoplasma debido a la erosión genética; por lo que el establecimiento, colección y conservación de germoplasma de camote constituyen una actividad importante; es por esto que el estudio de este cultivo es tan relavante.

El fitomejorador debe evaluar un enorme número de genotipos debido a la dificultad de encontrar una planta con todos los genes o atributos agronómicos que son necesarios (Chávez, 2002).

En la caracterización morfológica se evaluaron características de las hojas y de la raiz reservante, observando en los resultados una gran variabilidad genética, lo cual se expresó en las diferencias de formas del follaje y colores de pulpa. Se hizo también una evaluación de respuestas fenotipicas en campo: a) capacidad de prendimiento; b) vigor de follaje; c) grado de tuberización. Esta evaluación fue hecha en suelo árido-salino-bórico propio de la Irrigación La Yarada, en la ciudad de Tacna, con la finalidad de ver el nivel de adaptabilidad ante los efectos del estrés abiótico.

Los clones TN 95.041, TN 95.065, TN 95.075, TN 95.119 , TN 89.021, TN 95.660 y TN 95.649, asi como las variedades Atacama, Caplina, Comensal, Costanero y Salyboro, fueron los genotipos mejor adaptados a estas condiciones edáficas.

En la evaluación de grado de tuberización, los genotipos de mejor rendimiento fueron: TN 95.041, TN 95.065, TN 95.075, TN 95.119 y TN 95.649; por lo que es preciso continuar con la evaluación de estos genotipos hasta lograr la liberación de aquellos que muestren excelentes resultados bajo condiciones extremas, como es el caso de la toxicidad por boro, salinidad o sequía.

Se hizo también una evaluación importante, consistente en determinar el contenido de betacaroteno en la pulpa; de lo cual se obtuvieron resultados muy alentadores con los genotipos TN $95.075(3.64 \mathrm{mg} /$ $100 \mathrm{~g}$. de peso fresco), TN $95.667(3.63 \mathrm{mg} / 100 \mathrm{~g}$. de peso fresco), TN $95.065(2.91 \mathrm{mg} / 100 \mathrm{~g}$. de peso fresco), y el genotipo TN 95.041, con pulpa color naranja oscuro, pero al que no se le evaluó cuantitativamente el contenido de betacaroteno. Estos genotipos se presentan muy prometedores para la lucha contra las enfermedades de la vista en poblaciones de extrema pobreza, ya que el betacaroteno es un precursor de la vitamina $\mathrm{A}$.

Finalmente, se realizó una caracterización molecular mediante la técnica del AFLP, obteniendo información más certera que nos llevó a verificar que los genotipos en estudio son diferentes (no duplicados), ya que se hizo uso de la huella genética digital de cada genotipo, determinando las diferencias entre todos ellos.

La caracterización molecular hecha en el año 2001 se realizó con ocho combinaciones de primers (AGG) CAC, ACC/CTG, ACC/CAT, ACG/CAC, AAC/CTC, $A G C / C A G, A A C / C T G$ y ACC/CTG) para la amplificación con la técnica de AFLP. Luego de la tinción de los geles se realizó un análisis de presencia - ausencia en las bandas de cada gel, esto para determinar el polimorfismo que existe entre los genotipos de camote en estudio.

Las combinaciones de primers AAC/CTC, AAC/CTG yACC/CAT son las que proporcionan un mayor número de bandas polimórficas, por lo que estos primers ayudan a diferenciar genéticamente, y con mayor certeza, los genotipos de camote. En general, se observa que usando la técnica del AFLP se obtiene un porcentaje elevado de bandas polimórficas, lo cual hace eficiente esta técnica para la caracterización molecular. El dendograma producto de esta evaluación muestra 4 grupos de cultivares bien definidos, lo cual demuestra la utilidad del dendograma: determina individualidad de cada genotipo y a la vez determina relaciones filogenéticas entre todos lo genotipos en estudio.

En la caracterización molecular de las variedades de camote, en la que se incluyen los genotipos ST 87.030 y Yema de Huevo (Tambeño), hecha en el año 2004, se obtuvo un dendograma en los cuales los coeficientes de similaridad son menores que 1 , mostrando las diferencias entre los genotipos. Pero, asimismo, el dendograma mostró 3 grupos de cultivares bien definidos, mostrando asi las relaciones filogenéticas entre ellos.

Finalmente se hizo una caracterización molecular de 19 genotipos en los que se incluyen clones chinos y variedades de camote ya liberadas como testigos. En el dendograma que se,obtiene (Figua $N^{\circ} 05$ ), se puede observar que los genotipos poseen un coeficiente de similaridad menor que uno, tal como en los dos casos anteriores. Se evidencia una similaridad entre los genotipos 440158 y TN 95.041; así como entre los genotipos TN 92.024 y TN 95.537. Pero lo interesante de estos casos es que en ambas situaciones los genotipos provienen de progenitores distintos, por lo tanto esta garantizado que se trata de genotipos distintivos. Los demás genotipos si muestran coeficientesde similaridad menores de 0.90 , por lo que concluye que se trata de individuos diferentes y con una carga genética propia.

\section{CONCLUSIONES}

Las 3 evaluaciones hechas en los años 2001, 2003 y 2004 muestran un espectro de variabilidad genética significativa en la caracterización morfológica y molecular, ya que cada uno de los genotipos muestra un amplio rango de variabilidad geriética en los 
caracteres estudiados, en relación con los demás genotipos.

Los genotipos TN 95.065, TN 95.075, TN 95.119, TN 95.041 , TN 89.021, TN 95.660, TN 95.649, evidenciaron una gran adaptación a las condiciones de estrés abiótico, como ser la salinidad y la toxicidad por boro.

Los genotipos Salyboro, Costanero, TN 95.041, TN 95.075, TN 95.667, TN 91.012, TN 95.065, TN 95.596, presentan la pulpa de la raíz reservante color naranja, lo cual evidencia un alto contenido de betacaroteno. Por otro lado, el genotipo TN 95.119, con pulpa blanca, se mostró como un candidato a posteriores evaluaciones de mejoramiento.

La caracterización morfológica realizada en el año 2003 muestra un grupo de genotipos que no se adaptaron a las condiciones de estrés abiótico de La Yarada. Este dato es importante, pues es parte también de la variabilidad genética que existe entre los diversos genotipos en estudio.

La caracterización molecular con AFLP demostró que es una técnica muy útil para la obtención de marcadores polimórficos, los cuales ayudan a diferenciar un genotipo de otro. En el año 2001, utilizando 8 combinaciones de primers, se obtuvo un promedio de $78,09 \%$ de bandas polimórficas, porcentaje elevado y aceptable para poder emitir diagnósticos más certeros acerca de la afinidad genética entre genotipos y la individualidad de los mismos. El dendograma muestra 4 grupos de cultivares bien definidos, con coeficientes de similaridad menores que 1.

La caracterización molecular del año 2004 muestra un dendograma con coeficientes de similaridad también menores que $1 ;$ y, a la vez, muestra 3 grupos de cultivares bien definidos. Estos resultados evidencian la gran utilidad de los dendogramas.

\section{RECOMENDACIONES}

Se recomienda usar como progenitores en estudios genéticos posteriores a los genotipos TN 95.065 , TN 95.075 , TN 95.119, TN 95.041, TN 89.021, TN 95.660, TN 95.649, por ser los que mejores resultados han mostrado en las evaluaciones morfológicas y fenotípicas.

Es recomendable extender el cultivo del Clon Elite TN 95.075 para lograr su liberación, por ser un genotipo de excelentes condiciones agronómicas y con una característica importante: alto grado de betacaroteno en su pulpa.

Es recomendable continuar con la evaluación de los clones que también mostraron betacarotenos en sus pulpas, como son: TN 95.041, TN 95.065, TN 95.649y TN 95.667. Del mismo modo se recomienda continuar evaluando al clon TN 95.119, de pulpa blanca, pero de excelentes condiciones agronómicas.
Es recomendable extender la caracterización molecular de los genotipos estudiados de camote con otros marcadores, como el RFLP y SSR.

Es recomendable patentar las variedades mejoradas de camote por la Universidad Nacional Jorge Basadre Grohmann, usando los marcadores moleculares.

Es recomendable seguir trabajando en cruzamientos para obtener semilla botánica de camote y así iniciar nuevos procesos de evaluación, desde clones iniciales hasta lograr variedades mejoradas.

\section{REFERENCIA BIBL.IOGRÁFICA}

1. ALLARD, R.W. 1980. Princlpios de la mejora genética de las plantas. $4^{\circ} \mathrm{Ed}$. Editorial OMEGA, Barcelona, España

2. ARÉVALO, Nelly. 1995. "Evaluación Iniclal en Robiaciones heterogéneas de camote (Ipomoea batatas L.) en Tacna". Revista Ciencia y Desarrollo - COIN, UNJBG. No 01. Tacna, Perú.

3. BLEEKER, M.; M. Reijans; M. Hornes; J. Kuiper. 1997. AFLP Analysis System. Molecular Research Center. Life Technologies. Lincoln Center Drive. California - USA.

4. BONILLA, Nevio. Caracterización molecular de genotipos de papa mediante el uso de AFLPs. Asociación Latinoamericana de la Papa (ALAP). Centro Internacional de la Papa. Lima, Perú.

5. BUFFONE, Frank; R. La Bonte. 1996. "Random Ampliflled Polymorphic DNA Markers in Sweepotato infected with Fusarium lateritium". HortScience, Vol.31; Número 7, Lousiana State, USA. 6. CALZADA, Benza. 1982. Métodos estadisticos para la investigación. 5ta. Ed. Edit. Milagros, Lima - Perú.

7. CIP 1995. Breeding Sweetpotato for adaptation to Arid and Salin Solls. Circular del Centro Internacional de la Papa. Lima, Perú.

8. CIP 2000. Pathogen - tested sweetpotato cultivars for distribution cultivars. International Potato Center. Lima, Perú

9. CHAVEZ, René. 1990. Cultivo de raices y tubércuios. FCAG, UNJBG. Tacna, Perú.

10. CHÁVEZ, René. 1995. "Clones superiores de papa y camote adaptados a suelos árldo-sallno-bóricos: últimos avances". Revista Ciencia y Desarrollo - COIN, UNJBG N ${ }^{\circ} 02$, Tacna, Perú. 11. CHÁVEZ, René. 2002. Mejoramiento genétlco de plantas tuberiferas para zonas árido-sallno-bórlcas. Editorial ARTGRAPHICS. Tacna, Perú.

12. DOMINGUEZ. Aleiandro. 2003. Caracterización Molecular de Croton sp. (Sangre de Grado) de Uso Medicinal mediante Marcadores Moleculares RAPDs y AFLPs. Instituto de Biotecnología de la Universidad Nacional Agraria La Molina, Lima, Perú.

13. FERREIRA, M.E.; Grattapaglia, D. 1998. Introducción al Uso de Marcadores Moleculares en el Análisis Genético. Centro Nacional de Investigación de Brasilia, Brasil.

14. GALLO, Pedro. 2002. Introducción y Evaluación de Variedades Mejoradas de Camote (Ipomoea batatas L.) en las zonas árido-salinas del Norte de Chile. Arica, Chile.

15. GARCIA, Sandra. 2001. Empleo de Marcadores Moleculares AFLP en la determinación de la variabilidad genética de clones selectos de ajo (Allium sativum). Universidad Nacional Cuyo, Facultad de Ciencias Agrarias. Argentina.

16. HALE, Maynard; D. Orcutt. 1987. The Physiology of Plants Under Stress. United States of America.

17. PROMEGA. 1999. Technical Manual Silver Sequence DNA sequencing system. Introduction for use of products. USA

18. ROSSEL, Genoveva, A. Kriegner. 2000. From Latin American to Oceania: The Historlc Dispersal of Sweepotato Re-examined using AFLP. International Potato Center, CIP. Lima, Perú 19. STEEL Y TORRIE. Principios y procedimlentos de bloestadistica. $2^{\circ} \mathrm{Ed}$.

20. UNALM, Publicación: "Camota: Alimento Barato, Fuente de Energla y Carotenos". Universidad Nacional Agraria La Molina. Lima, Perú. www.lamolina.edu.pe/Investigacion/programa/camote/

21. VERA, Nadiz. 2001. Caracterización morfológica y molecular de 25 genotipos de camote (Ipomoea batatas L.) adaptadas a zonas desérticas. Tesis para optar el título de Blólogo. Microbiólogo. Universidad Nacional Jorge Basadre Grohmann. Tacna, Perú. 


\section{ANEXOS}

Follaje de diversos genotipos de camote (Ipomoea batatas L.) Se aprecia la varibilidad genética en la forma de las hojas
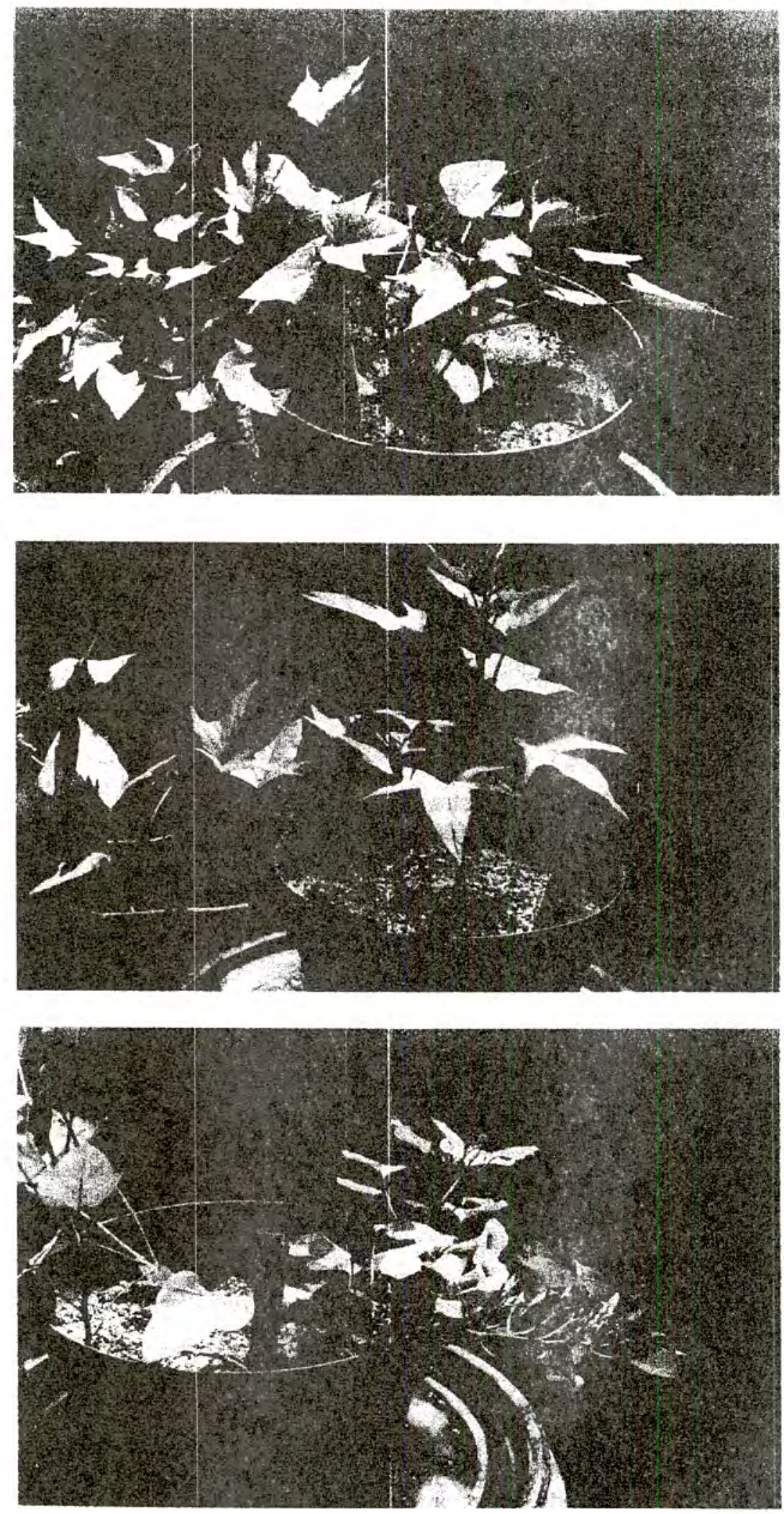

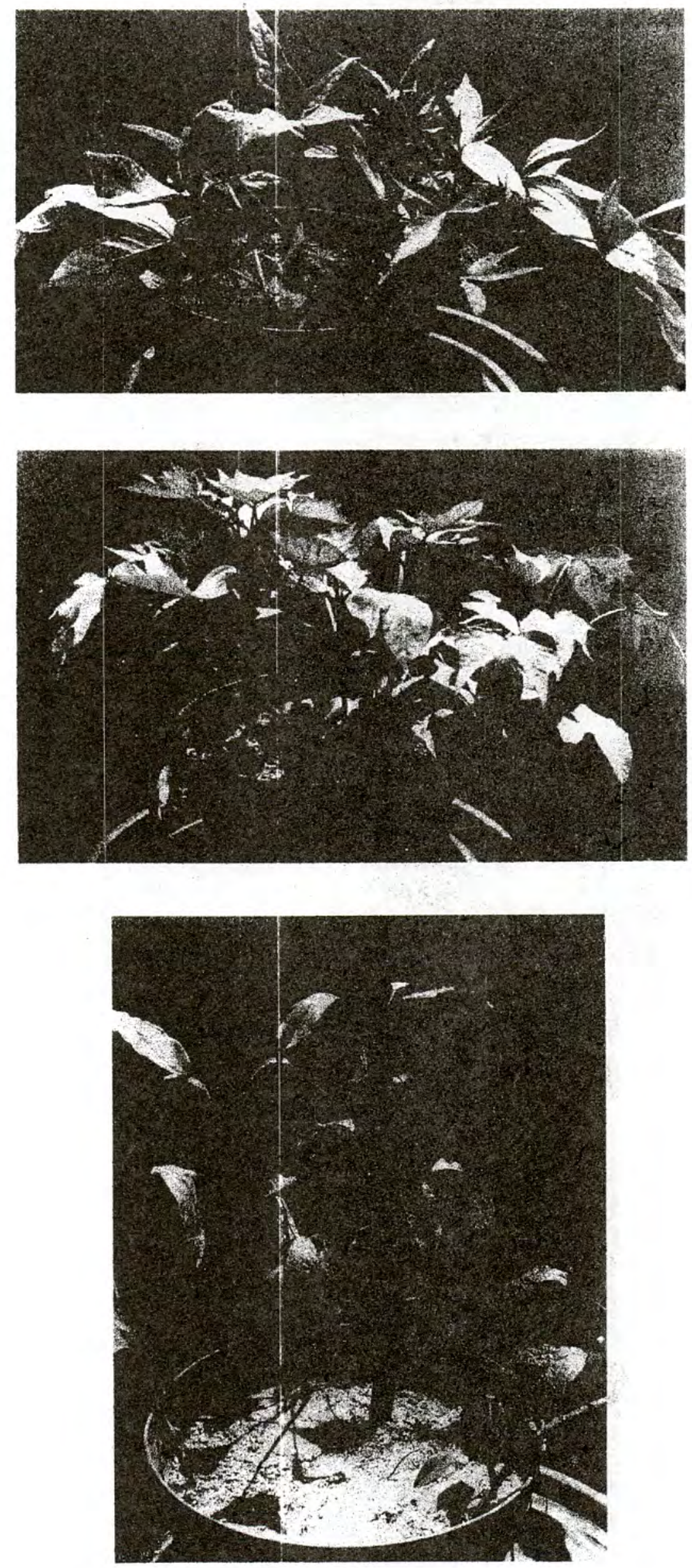
Follaje de diversos genotipos de camote (Ipomoea batatasL.) Se aprecia la varibilidad genética en la forma de la hojas
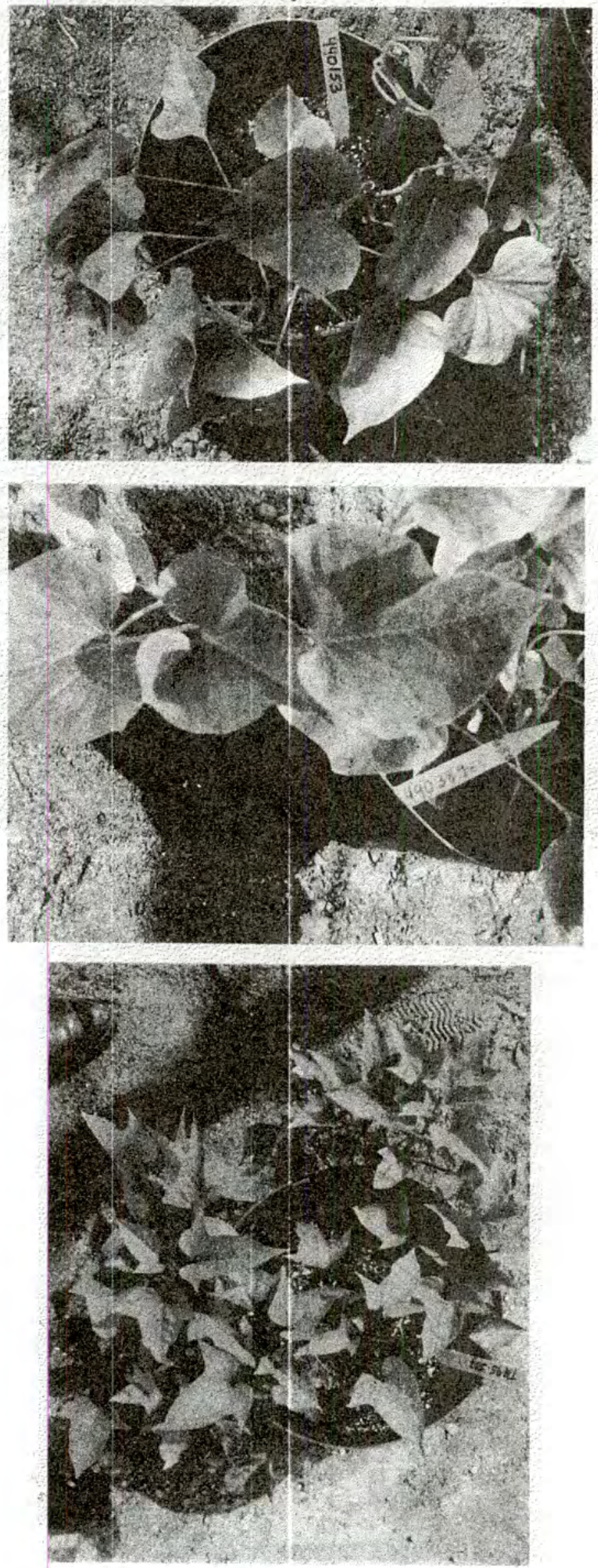

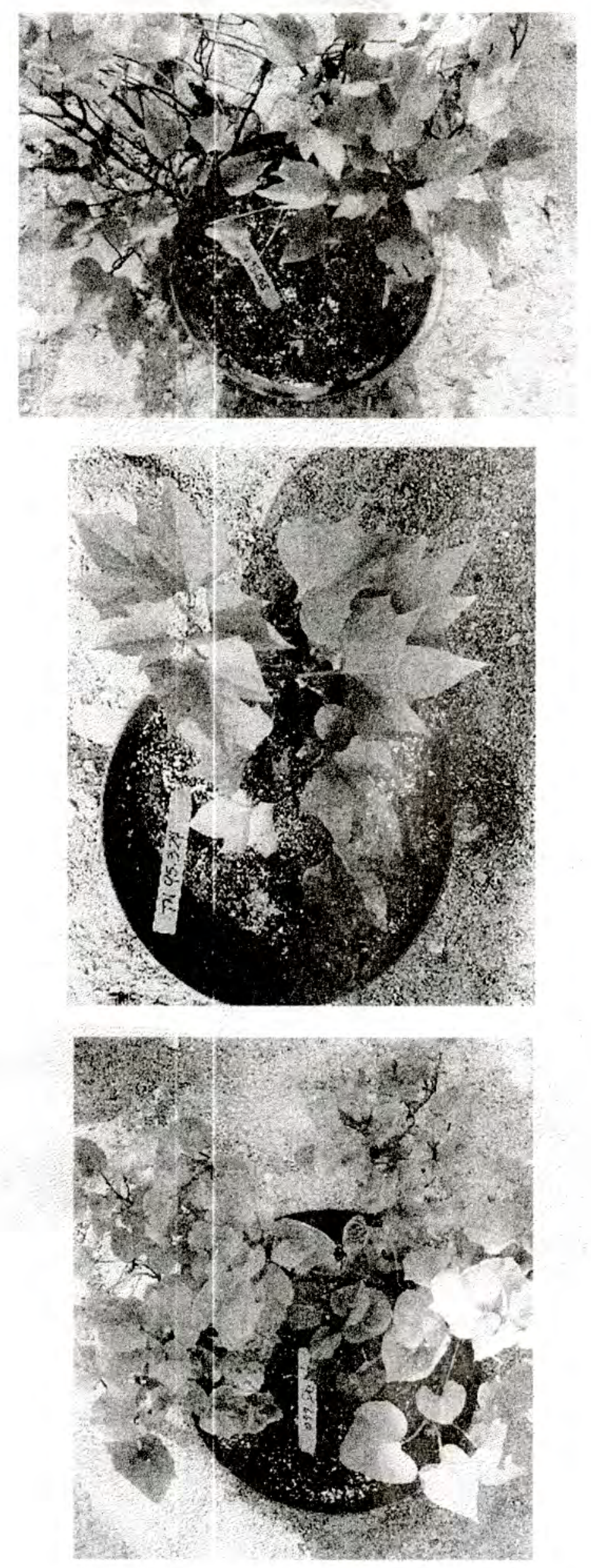
Follaje de diversos genotipos de camote (Ipomoea batatas $L$.) Se aprecia la varibilidad genética en la forma de las hojas

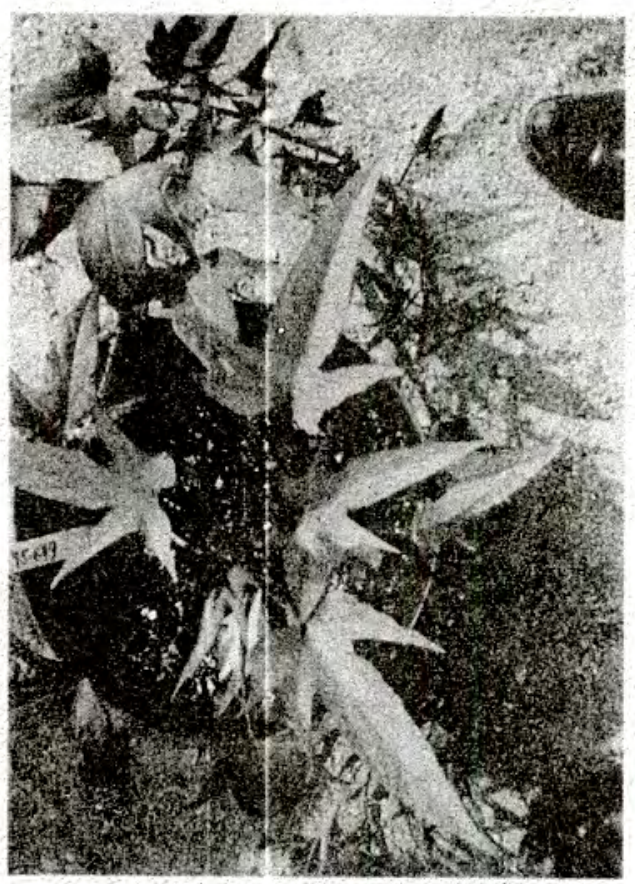

Los clones TN95.041 y TN 95.075 poseen un elevado contenido de betacaroteno, lo que les da un intenso color naranja a la pulpa
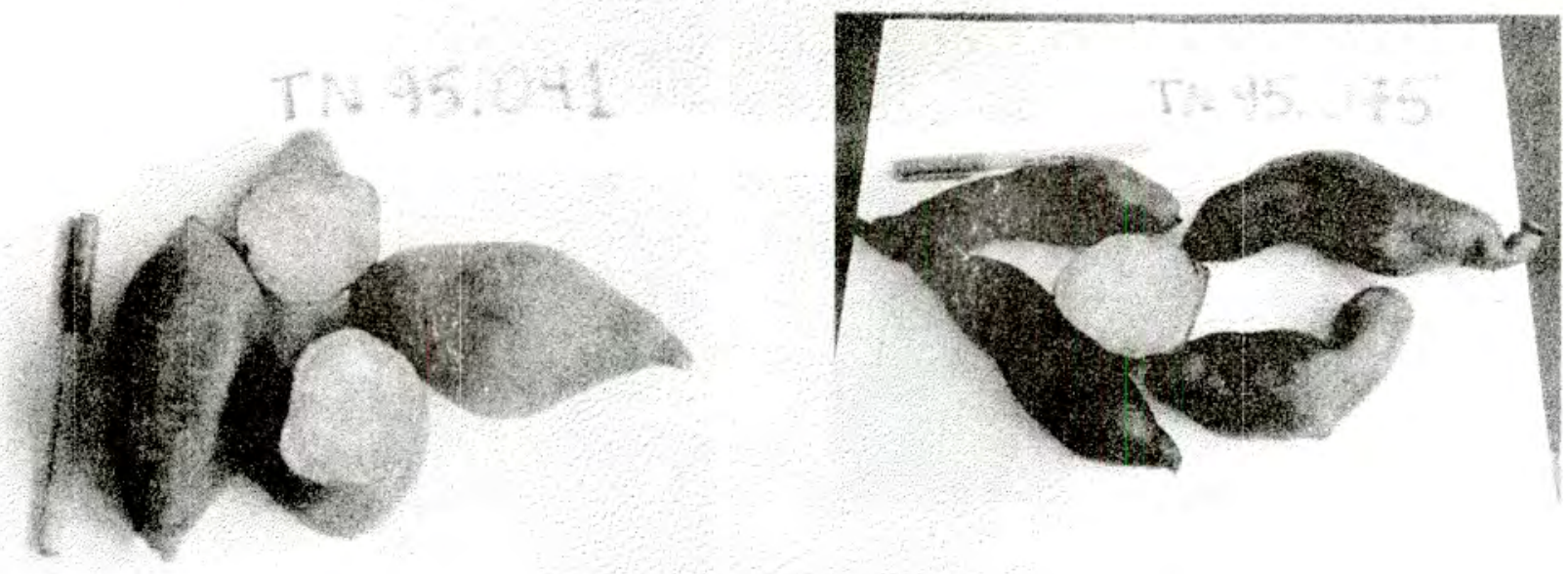